\title{
The stochastic field of aggregate utilities and its saddle conjugate.
}

\author{
P. Bank \\ Technische Universität Berlin \\ Institut für Mathematik \\ Straße des 17. Juni 135, 10623 Berlin, Germany \\ (bank@math.tu-berlin.de) \\ D. Kramkov* \\ Carnegie Mellon University, \\ Department of Mathematical Sciences, \\ 5000 Forbes Avenue, Pittsburgh, PA, 15213-3890, US \\ (kramkov@cmu.edu) \\ July 19, 2018
}

\begin{abstract}
We describe the sample paths of the stochastic field $F=F_{t}(v, x, q)$ of aggregate utilities parameterized by Pareto weights $v$ and total cash amounts $x$ and stocks' quantities $q$ in an economy. We also describe the sample paths of the stochastic field $G=G_{t}(u, y, q)$, which is conjugate to $F$ with respect to the saddle arguments $(v, x)$, and obtain various conjugacy relations between these stochastic fields. The results of this paper play a key role in our study in [1], [2], and [3] of a continuous-time price impact model.
\end{abstract}

*The author also holds a part-time position at the University of Oxford. This research was supported in part by the Carnegie Mellon-Portugal Program and by the Oxford-Man Institute for Quantitative Finance at the University of Oxford. 
Keywords: Envelope theorem, equilibrium, utility indifference prices, Pareto allocation, price impact model, risk-aversion, risk-tolerance, saddle function, stochastic field.

MSC: 52A41, 60G60, 91G10, 91G20.

JEL Classification: G11, G12, G13, C61.

\section{Contents}

1 Setup and motivation 3

2 Conjugate spaces of saddle functions 7

2.1 The spaces $\mathbf{F}^{1}$ and $\mathbf{F}^{2} \ldots \ldots \ldots \ldots 7$

2.2 The spaces $\mathbf{G}^{1}$ and $\mathbf{G}^{2} \ldots \ldots \ldots . \ldots . \ldots 8$

2.3 Conjugacy relations between $\mathbf{F}^{1}$ and $\mathbf{G}^{1} \ldots \ldots$. . . . . . . 10

2.3.1 Proof of Theorem 2.2 . . . . . . . . . . . . . . . . . 10

2.4 Conjugacy relations between $\mathbf{F}^{2}$ and $\mathbf{G}^{2} \ldots \ldots . . . . . .22$

2.4.1 Proof of Theorem 2.10 . . . . . . . . . . . . . 23

2.5 Stability under convergence . . . . . . . . . . . . . 28

2.5.1 Proofs of Theorems 2.14 and 2.15 . . . . . . . . . 28

2.6 Additional conjugacy relations . . . . . . . . . . . . . . . 32

2.6.1 Proof of Theorem 2.18 . . . . . . . . . . . . . . 34

2.7 The spaces $\widetilde{\mathbf{F}}^{1}, \widetilde{\mathbf{G}}^{1}$ and $\widetilde{\mathbf{F}}^{2}(c), \widetilde{\mathbf{G}}^{2}(c) \ldots \ldots . \ldots 36$

3 Aggregate utility function 37

3.1 Proofs of Theorems 3.1 and $3.2 \ldots \ldots$. . . . . . . . . . 38

4 Stochastic field of aggregate utilities and its conjugate 42

4.1 Proofs of Theorems 4.1 and $4.2 \ldots \ldots$. . . . . . . . . 45

A An envelope theorem for saddle functions 50

B Integrability of saddle random fields $\quad 52$

C Stochastic fields of martingales 56 


\section{Setup and motivation}

Let $u_{m}=u_{m}(x), m=1, \ldots, M$, be functions on the real line $\mathbf{R}$ satisfying

Assumption 1.1. Each function $u_{m}$ is strictly concave, strictly increasing, continuously differentiable, and such that

$$
\lim _{x \rightarrow \infty} u_{m}(x)=0
$$

The normalization to zero in (1.1) is added only for notational convenience. From Assumption 1.1 we clearly deduce that

$$
\lim _{x \rightarrow-\infty} u_{m}(x)=-\infty
$$

Many of our results are derived under the additional condition which, in particular, implies the boundedness of $u_{m}$ from above.

Assumption 1.2. Each function $u_{m}$ is twice continuously differentiable and, for some constant $c>0$,

$$
\frac{1}{c} \leq a_{m}(x) \triangleq-\frac{u_{m}^{\prime \prime}(x)}{u_{m}^{\prime}(x)} \leq c, \quad x \in \mathbf{R} .
$$

In the model of price impact discussed below, the functions $\left(u_{m}\right)$ and $\left(a_{m}\right)$ describe agents' utilities and absolute risk-aversions. From Assumptions 1.1 and 1.2 we deduce that

$$
\frac{1}{c} \leq-\frac{u_{m}^{\prime}(x)}{u_{m}(x)} \leq c, \quad x \in \mathbf{R}
$$

Denote by $r=r(v, x)$ the $v$-weighted sup-convolution:

$$
r(v, x) \triangleq \max _{x^{1}+\cdots+x^{M}=x} \sum_{m=1}^{M} v^{m} u_{m}\left(x^{m}\right), \quad(v, x) \in(0, \infty)^{M} \times \mathbf{R} .
$$

The properties of this function are collected in Section 3. In particular, for every $v \in(0, \infty)^{M}$, the function $r(v, \cdot)$ on $\mathbf{R}$ satisfies same Assumptions 1.1 and 1.2 as each $u_{m}$. As usual, in financial economics, we call $r=r(v, x)$ the aggregate utility function. 
Let $\Sigma_{0}$ and $\psi=\left(\psi^{j}\right)_{j=1, \ldots, J}$ be random variables on a complete filtered probability space $\left(\Omega, \mathscr{F}_{T},\left(\mathscr{F}_{t}\right)_{0 \leq t \leq T}, \mathbb{P}\right)$ with a finite maturity $T$. Denote

$$
\Sigma(x, q) \triangleq \Sigma_{0}+x+\langle q, \psi\rangle=\Sigma_{0}+x+\sum_{j=1}^{J} q^{j} \psi^{j}, \quad(x, q) \in \mathbf{R} \times \mathbf{R}^{J}
$$

and assume that

$$
\mathbb{E}[r(v, \Sigma(x, q))]>-\infty, \quad(v, x, q) \in \mathbf{A}
$$

where

$$
\mathbf{A} \triangleq(0, \infty)^{M} \times \mathbf{R} \times \mathbf{R}^{J}
$$

The main results of the paper, Theorems 4.1 and 4.2, describe the sample paths of the stochastic fields $F=F_{t}(a)$ and $G=G_{t}(b)$ given, for $t \in[0, T]$, by

$$
\begin{aligned}
& F_{t}(a) \triangleq \mathbb{E}\left[r(v, \Sigma(x, q)) \mid \mathscr{F}_{t}\right], \quad a=(v, x, q) \in \mathbf{A}, \\
& G_{t}(b) \triangleq \sup _{v \in(0, \infty)^{M}} \inf _{x \in \mathbf{R}}\left[\langle v, u\rangle+x y-F_{t}(v, x, q)\right], \quad b=(u, y, q) \in \mathbf{B},
\end{aligned}
$$

where

$$
\mathbf{B} \triangleq(-\infty, 0)^{M} \times(0, \infty) \times \mathbf{R}^{J}
$$

In particular, Theorem 4.1 shows that these fields have versions which are differentiable in their spatial arguments and RCLL with respect to time, while Theorem 4.2 provides uniform estimates for their spatial second order derivatives depending only on the risk-aversion bound of Assumption 1.2. In view of its construction, we call $F$ the stochastic field of aggregate utilities.

Of course, the basic regularity properties of $r=r(v, x)$ are well-known, albeit mostly stated for utility functions on the positive half line. We refer to Dana [4], Dana and Le Van [5] as well as Karatzas et al. [7] and the references therein for pertaining results, e.g., on the differentiability in $x$ and on the sensitivities with respect to $v$. To the best of our knowledge though, the filtered version of the induced expected utilities as described by the field $F$ has not been investigated in depth before. In particular, its structure as an RCLL process in a space of saddle functions seems to be new. We also could 
not find a reference where the saddle conjugate $G$ would be analyzed or made used of.

Our work is motivated by the study of a financial model with price impact; see the accompanying papers [1], [2], and [3]. In this model, $M$ market makers quote utility indifference prices for $J$ stocks to a large investor. The market makers' preferences are specified by the utility functions $\left(u_{m}\right)_{m=1, \ldots, M}$ for terminal wealth. Their initial total endowment is given by $\Sigma_{0}$. The stocks pay the terminal dividends $\psi=\left(\psi^{j}\right)_{j=1, \ldots, J}$.

If, as a result of trading with a large investor up to time $t$, the market makers acquire a cash amount $x$ and quantities of stocks $q=\left(q^{j}\right)$, then their total endowment becomes $\Sigma(x, q)$. The model assumes that $\Sigma(x, q)$ is distributed among the market makers in the form of a Pareto optimal allocation $\pi(a)=\left(\pi^{m}(a)\right)$ which, for $a=(v, x, q) \in \mathbf{A}$, is given by

$$
v^{m} u_{m}^{\prime}\left(\pi^{m}(a)\right)=\frac{\partial r}{\partial x}(v, \Sigma(x, q)), \quad m=1, \ldots, M .
$$

Here the Pareto weight $v \in(0, \infty)^{M}$ is unique up to a multiplication on a positive constant. A trade at time $t$ in $\Delta q$ stocks results in the new Pareto optimal allocation $\pi(a+\Delta a)=\pi(x+\Delta x, q+\Delta q, v+\Delta v)$ determined by the condition of utility indifference:

$$
\mathbb{E}\left[u_{m}\left(\pi^{m}(a)\right) \mid \mathscr{F}_{t}\right]=\mathbb{E}\left[u_{m}\left(\pi^{m}(a+\Delta a)\right) \mid \mathscr{F}_{t}\right], \quad m=1, \ldots, M .
$$

More generally, given an $\mathbf{R}^{J}$-valued demand process $Q=\left(Q_{t}^{j}\right)$ for stocks, the model's evolution is described by the processes $X=\left(X_{t}\right)$, of cash amounts, and $V=\left(V_{t}^{m}\right)$, of Pareto weights, solving the equation:

$$
U_{t}^{m}\left(A_{t}\right)=U_{0}^{m}\left(A_{0}\right)+\int_{0}^{t} U^{m}\left(A_{s}, d s\right), \quad t \in[0, T], m=1, \ldots, M .
$$

Here $A \triangleq(V, X, Q)$ is a predictable process with values in $\mathbf{A}$, the stochastic field

$$
U_{t}^{m}(a)=U^{m}(a, t) \triangleq \mathbb{E}\left[u_{m}\left(\pi^{m}(a)\right) \mid \mathscr{F}_{t}\right], \quad a \in \mathbf{A},
$$

represents the expected utilities for the $m$ th market maker, and the nonlinear stochastic integral of $A$ against this field is defined as in Section 3.2 of Kunita [8]. In particular, if $U^{m}$ admits the integral representation

$$
U_{t}^{m}(a)=U_{0}^{m}(a)+\int_{0}^{t} K_{s}^{m}(a) d B_{s}
$$


with respect to a $d$-dimensional Brownian motion $B$ such that the $d$-dimensional stochastic field $K^{m}=K_{s}^{m}(a)$ has continuous sample paths with respect to $a$, then

$$
\int_{0}^{t} U^{m}\left(A_{s}, d s\right) \triangleq \int_{0}^{t} K_{s}^{m}\left(A_{s}\right) d B_{s} .
$$

If we restrict $V$ to take values in the interior of the simplex:

$$
\mathbf{S}^{M} \triangleq\left\{w \in(0,1)^{M}: \sum_{m=1}^{M} w^{m}=1\right\},
$$

then, for given $Q$, the model's evolution is well-defined if (1.9) has a unique solution $(X, V)$. The mathematical challenge is then to specify the conditions when this is true.

A natural idea in the study of (1.9) is to substitute $U$ for $(X, V)$ since then this equation takes a familiar "explicit" form. The related construction of the stochastic fields $X=\left(X_{t}(u, q)\right)$ and $V=\left(V_{t}^{m}(u, q)\right)$ inverse to $U=$ $\left(U_{t}^{m}(x, u, q)\right)$, that is, such that

$$
U_{t}^{m}\left(X_{t}(u, q), V_{t}(u, q), q\right)=u^{m}, \quad m=1, \ldots, M,
$$

is greatly simplified by the observation that $U$ is the $v$-gradient of $F$ :

$$
U_{t}^{m}(a)=\frac{\partial F_{t}}{\partial v^{m}}(a), \quad a=(v, x, q), m=1, \ldots, M .
$$

The theory of conjugate saddle functions, see Part VII of Rockafellar [10], then allows us to express $X$ and $V$ in terms of the partial derivatives of $G$ :

$$
\begin{aligned}
X_{t}(u, q) & =\frac{\partial G_{t}}{\partial y}(u, 1, q)=G_{t}(u, 1, q), \\
V_{t}^{m}(u, q) & =\frac{\partial G_{t}}{\partial u^{m}} \frac{1}{\sum_{l=1}^{M} \frac{\partial G_{t}}{\partial u^{l}}}(u, 1, q) .
\end{aligned}
$$

Thus, the properties of the stochastic fields $U, X$, and $V$ follow from those of $F$ and $G$, motivating the study of the latter.

We refer the reader to [1] and [2] for more details on the price impact model and to [3] for convenient sufficient conditions guaranteeing unique solvability of (1.9). These papers use extensively the results of the current study and explain the economic background as well as related work. 
The paper is organized as follows. The appropriate conjugate spaces of saddle functions are defined and studied in Section 2. In Section 4 these spaces are shown to contain the sample paths of the stochastic fields $F_{t}=$ $F_{t}(a)$ and $G_{t}=G_{t}(b)$. The proofs of the main theorems in Section 4 also rely on the properties of the function $r=r(v, x)$ given in Section 3, on the "envelope" theorem for saddle functions stated in Appendix A, and on the criteria for sample paths of random and stochastic fields with values in saddle functions presented in Appendices B and C.

\section{Conjugate spaces of saddle functions}

In this section we study the conjugate spaces of saddle functions which later are shown to support the sample paths of the stochastic fields $F=F_{t}(a)$ and $G=G_{t}(b)$.

Recall some standard notations. For a function $f=f(x, y)$, where $x \in$ $\mathbf{R}^{n}$ and $y \in \mathbf{R}^{m}$, we denote by $\frac{\partial f}{\partial x} \triangleq\left(\frac{\partial f}{\partial x^{1}}, \ldots, \frac{\partial f}{\partial x^{n}}\right)$ the vector of partial derivatives with respect to $x$ and by $\nabla f \triangleq\left(\frac{\partial f}{\partial x}, \frac{\partial f}{\partial y}\right)$ its gradient. For a set $A \subset \mathbf{R}^{d}$, the notations $\partial A$ and $\operatorname{cl} A$ stand for, respectively, the boundary and the closure. For $x, y \in \mathbf{R}^{d}$ denote $\langle x, y\rangle \triangleq \sum_{i=1}^{d} x^{i} y^{i}$ and $|x| \triangleq \sqrt{\langle x, x\rangle}$, the Euclidean scalar product and the norm.

\subsection{The spaces $\mathrm{F}^{1}$ and $\mathrm{F}^{2}$}

Recall the construction of the parameter set $\mathbf{A}$ from (1.6) and the notation $\mathbf{S}^{M}$ from (1.10) for the interior of the simplex in $\mathbf{R}^{M}$. We shall often decompose $a \in \mathbf{A}$ as $a=(v, x, q)$, where $v \in(0, \infty)^{M}, x \in \mathbf{R}$, and $q \in \mathbf{R}^{J}$.

For a function $f: \mathbf{A} \rightarrow(-\infty, 0)$ define the following conditions:

(F1) The function $f$ is continuously differentiable on $\mathbf{A}$.

(F2) For every $(x, q) \in \mathbf{R} \times \mathbf{R}^{J}$, the function $f(\cdot, x, q)$ is positively homogeneous:

$$
f(z v, x, q)=z f(v, x, q) \text {, for all } z>0 \text { and } v \in(0, \infty)^{M},
$$

and strictly decreasing on $(0, \infty)^{M}$. Moreover, if $M>1$ then $f(\cdot, x, q)$ is strictly convex on $\mathbf{S}^{M}$ and

$$
\lim _{n \rightarrow \infty} f\left(w_{n}, x, q\right)=0,
$$


for every sequence $\left(w_{n}\right)_{n \geq 1}$ in $\mathbf{S}^{M}$ converging to a boundary point of $\mathbf{S}^{M}$.

(F3) For every $v \in(0, \infty)^{M}$, the function $f(v, \cdot, \cdot)$ is concave on $\mathbf{R} \times \mathbf{R}^{J}$.

(F4) For every $(v, q) \in(0, \infty)^{M} \times \mathbf{R}^{J}$, the function $f(v, \cdot, q)$ is strictly concave and strictly increasing on $\mathbf{R}$ and

$$
\lim _{x \rightarrow \infty} f(v, x, q)=0
$$

(F5) The function $f$ is twice continuously differentiable on $\mathbf{A}$ and, for every $a=(v, x, q) \in \mathbf{A}$,

$$
\frac{\partial^{2} f}{\partial x^{2}}(a)<0
$$

and the matrix $A(f)(a)=\left(A^{l m}(f)(a)\right)_{l, m=1, \ldots, M}$ given by

$$
A^{l m}(f)(a) \triangleq \frac{v^{l} v^{m}}{\frac{\partial f}{\partial x}}\left(\frac{\partial^{2} f}{\partial v^{l} \partial v^{m}}-\frac{1}{\frac{\partial^{2} f}{\partial x^{2}}} \frac{\partial^{2} f}{\partial v^{l} \partial x} \frac{\partial^{2} f}{\partial v^{m} \partial x}\right)(a),
$$

has full rank.

We now define the families of functions:

$$
\begin{aligned}
& \mathbf{F}^{1} \triangleq\{f: \mathbf{A} \rightarrow(-\infty, 0):(\mathrm{F} 1)-(\mathrm{F} 4) \text { hold }\} \\
& \mathbf{F}^{2} \triangleq\left\{f \in \mathbf{F}^{1}: \text { (F5) holds }\right\} .
\end{aligned}
$$

Remark 2.1. Slightly abusing notations we shall use the same symbols $\mathbf{F}^{i}$, $i=1,2$, for the families of functions $f=f(v, x)$ on $(0, \infty)^{M} \times \mathbf{R}$ whose natural extensions $\widetilde{f}(v, x, q) \triangleq f(v, x)$ to functions on $\mathbf{A}$ belong to $\mathbf{F}^{i}$. Note that, in this case, (F3) follows trivially from (F4). A similar convention will also be used for other spaces of functions introduced below.

\subsection{The spaces $\mathrm{G}^{1}$ and $\mathrm{G}^{2}$}

Recall the construction of the parameter set $\mathbf{B}$ in (1.7). We shall often decompose $b \in \mathbf{B}$ as $b=(u, y, q)$, where $u \in(-\infty, 0)^{M}, y \in(0, \infty)$, and $q \in \mathbf{R}^{J}$.

For a function $g: \mathbf{B} \rightarrow \mathbf{R}$ define the following conditions: 
(G1) The function $g$ is continuously differentiable on $\mathbf{B}$.

(G2) For every $(y, q) \in(0, \infty) \times \mathbf{R}^{J}$, the function $g(\cdot, y, q)$ is strictly increasing and strictly convex on $(-\infty, 0)^{M}$. Moreover,

(a) If $\left(u_{n}\right)_{n \geq 1}$ is a sequence in $(-\infty, 0)^{M}$ converging to 0 , then

$$
\lim _{n \rightarrow \infty} g\left(u_{n}, y, q\right)=\infty .
$$

(b) If $\left(u_{n}\right)_{n \geq 1}$ is a sequence in $(-\infty, 0)^{M}$ converging to a boundary point of $(-\infty, 0)^{M}$, then

$$
\lim _{n \rightarrow \infty}\left|\frac{\partial g}{\partial u}\left(u_{n}, y, q\right)\right|=\infty .
$$

(c) If $M>1$ and $\left(u_{n}\right)_{n \geq 1}$ is a sequence in $(-\infty, 0)^{M}$ such that

$$
\limsup _{n \rightarrow \infty} u_{n}^{m}<0 \text { for all } m=1, \ldots, M
$$

and

$$
\lim _{n \rightarrow \infty} u_{n}^{m_{0}}=-\infty \text { for some } m_{0} \in\{1, \ldots, M\},
$$

then

$$
\lim _{n \rightarrow \infty} g\left(u_{n}, y, q\right)=-\infty .
$$

(G3) For every $y \in(0, \infty)$, the function $g(\cdot, y, \cdot)$ is convex on $(-\infty, 0)^{M} \times \mathbf{R}^{J}$.

(G4) For every $(u, q) \in(-\infty, 0)^{M} \times \mathbf{R}^{J}$, the function $g(u, \cdot, q)$ is positively homogeneous, that is,

$$
g(u, y, q)=y g(u, 1, q), \quad y>0 .
$$

(G5) The function $g$ is twice continuously differentiable on $\mathbf{B}$ and, for every $b=(u, y, q) \in \mathbf{B}$, the matrix $B(g)(b)=\left(B^{l m}(g)(b)\right)_{l, m=1, \ldots, M}$ given by

$$
B^{l m}(g)(b) \triangleq \frac{y}{\frac{\partial g}{\partial u^{l}} \frac{\partial g}{\partial u^{m}}} \frac{\partial^{2} g}{\partial u^{l} \partial u^{m}}(b)
$$

has full rank.

We define the families of functions

$$
\begin{aligned}
& \mathbf{G}^{1} \triangleq\{g: \mathbf{B} \rightarrow \mathbf{R}:(\mathrm{G} 1)-(\mathrm{G} 4) \text { hold }\}, \\
& \mathbf{G}^{2} \triangleq\left\{g \in \mathbf{G}^{1}: \text { (G5) holds }\right\} .
\end{aligned}
$$




\subsection{Conjugacy relations between $\mathrm{F}^{1}$ and $\mathrm{G}^{1}$}

The following theorem establishes the key conjugacy relations of this paper.

Theorem 2.2. A function $f: \mathbf{A} \rightarrow(-\infty, 0)$ belongs to $\mathbf{F}^{1}$ if and only if there is $g \in \mathbf{G}^{1}$ which is conjugate to $f$ in the sense that, for every $(u, y, q) \in$ B,

$$
\begin{aligned}
g(u, y, q) & =\sup _{v \in(0, \infty)^{M}} \inf _{x \in \mathbf{R}}[\langle v, u\rangle+x y-f(v, x, q)] \\
& =\inf _{x \in \mathbf{R}} \sup _{v \in(0, \infty)^{M}}[\langle v, u\rangle+x y-f(v, x, q)]
\end{aligned}
$$

and, for every $(v, x, q) \in \mathbf{A}$,

$$
\begin{aligned}
f(v, x, q) & =\sup _{u \in(-\infty, 0)^{M}} \inf _{y \in(0, \infty)}[\langle v, u\rangle+x y-g(u, y, q)] \\
& =\inf _{y \in(0, \infty)} \sup _{u \in(-\infty, 0)^{M}}[\langle v, u\rangle+x y-g(u, y, q)] .
\end{aligned}
$$

The minimax values in (2.12) and (2.13) are attained at unique saddle points and, for every fixed $q \in \mathbf{R}^{J}$, the following conjugacy relationships between $(v, x) \in(0, \infty)^{M} \times \mathbf{R}$ and $(u, y) \in(-\infty, 0)^{M} \times(0, \infty)$ are equivalent:

1. Given $(u, y)$, the minimax values in (2.12) are attained at $(v, x)$.

2. Given $(v, x)$, the minimax values in (2.13) are attained at $(u, y)$.

3. We have $x=\frac{\partial g}{\partial y}(u, y, q)=g(u, 1, q)$ and $v=\frac{\partial g}{\partial u}(u, y, q)$.

4. We have $y=\frac{\partial f}{\partial x}(v, x, q)$ and $u=\frac{\partial f}{\partial v}(v, x, q)$.

Moreover, in this case, $f(v, x, q)=\langle u, v\rangle, g(u, y, q)=x y$, and

$$
\frac{\partial g}{\partial q}(u, y, q)=-\frac{\partial f}{\partial q}(v, x, q) \text {. }
$$

\subsubsection{Proof of Theorem 2.2}

The proof relies on the theory of saddle functions presented in Part VII of the classical book [10] by Rockafellar. To simplify notations we omit the dependence on $q$, where it is not important, and then interpret the classes $\mathbf{F}^{1}$ and $\mathbf{G}^{1}$ in the sense of Remark 2.1. 
Lemma 2.3. Let $f=f(v, x):(0, \infty)^{M} \times \mathbf{R} \rightarrow(-\infty, 0)$ be in $\mathbf{F}^{1}$. Then there exists a continuously differentiable $g=g(u, y):(-\infty, 0)^{M} \times(0, \infty) \rightarrow$ $\mathbf{R}$, which is conjugate to $f$ in the sense that, for every $u \in(-\infty, 0)^{M}$ and $y \in(0, \infty)$,

$$
\begin{aligned}
g(u, y) & =\sup _{v \in(0, \infty)^{M}} \inf _{x \in \mathbf{R}}[\langle v, u\rangle+x y-f(v, x)] \\
& =\inf _{x \in \mathbf{R}} \sup _{v \in(0, \infty)^{M}}[\langle v, u\rangle+x y-f(v, x)],
\end{aligned}
$$

and, for every $v \in(0, \infty)^{M}$ and $x \in \mathbf{R}$,

$$
\begin{aligned}
f(v, x) & =\sup _{u \in(-\infty, 0)^{M}} \inf _{y \in(0, \infty)}[\langle v, u\rangle+x y-g(u, y)], \\
& =\inf _{y \in(0, \infty)} \sup _{u \in(-\infty, 0)^{M}}[\langle v, u\rangle+x y-g(u, y)] .
\end{aligned}
$$

Moreover, the minimax values in (2.15) and (2.16) are attained at unique saddle points.

Proof. To facilitate the references to Section 37 in [10] we define $f$ on the whole Euclidean space $\mathbf{R}^{M+1}$ by setting its values outside of $(0, \infty)^{M} \times \mathbf{R}$ as

$$
f(v, x)=\left\{\begin{array}{rl}
0, & v \in \partial \mathbf{R}_{+}^{M} \\
\infty, & v \notin \mathbf{R}_{+}^{M}
\end{array}, \quad x \in \mathbf{R},\right.
$$

where $\mathbf{R}_{+}^{M} \triangleq[0, \infty)^{M}$. By (2.1) and (2.2), after this extension $f$ becomes a closed saddle function (according to the definition in Section 34 of [10]) with effective domain

$$
\operatorname{dom} f \triangleq \operatorname{dom}_{1} f \times \operatorname{dom}_{2} f=\mathbf{R}_{+}^{M} \times \mathbf{R},
$$

where

$$
\begin{aligned}
& \operatorname{dom}_{1} f \triangleq\left\{v \in \mathbf{R}^{M}: f(v, x)<\infty, \quad \forall x \in \mathbf{R}\right\}=\mathbf{R}_{+}^{M} \\
& \operatorname{dom}_{2} f \triangleq\left\{x \in \mathbf{R}: f(v, x)>-\infty, \quad \forall v \in \mathbf{R}^{M}\right\}=\mathbf{R} .
\end{aligned}
$$


Using the extended version of $f$ we introduce the saddle functions

$$
\begin{aligned}
& \underline{g}(u, y) \triangleq \sup _{v \in \mathbf{R}^{M}} \inf _{x \in \mathbf{R}}[\langle v, u\rangle+x y-f(v, x)] \\
& =\sup _{v \in \mathbf{R}_{+}^{M}} \inf _{x \in \mathbf{R}}[\langle v, u\rangle+x y-f(v, x)], \\
& \bar{g}(u, y) \triangleq \inf _{x \in \mathbf{R}} \sup _{v \in \mathbf{R}^{M}}[\langle v, u\rangle+x y-f(v, x)] \\
& =\inf _{x \in \mathbf{R}} \sup _{v \in \mathbf{R}_{+}^{M}}[\langle v, u\rangle+x y-f(v, x)]
\end{aligned}
$$

defined for $u \in \mathbf{R}^{M}$ and $y \in \mathbf{R}$ and taking values in $[-\infty, \infty]$. By the duality theory for conjugate saddle functions, see [10], Theorem 37.1 and Corollaries 37.1.1 and 37.1.2, the functions $g$ and $\bar{g}$ have a common effective domain, which we denote $C \times D$, and coincide on (int $C \times D) \cup(C \times \operatorname{int} D)$, where $\operatorname{int} A$ denotes the interior of a set $A$.

Hence, on (int $C \times D) \cup(C \times \operatorname{int} D)$ we can define a finite saddle function $g=g(u, y)$ such that

$$
\begin{aligned}
g(u, y) & =\sup _{v \in \mathbf{R}_{+}^{M}} \inf _{x \in \mathbf{R}}[\langle v, u\rangle+x y-f(v, x)] \\
& =\inf _{x \in \mathbf{R}_{v \in \mathbf{R}_{+}^{M}}} \sup _{v}[\langle v, u\rangle+x y-f(v, x)] .
\end{aligned}
$$

Moreover, from the same Theorem 37.1 and Corollaries 37.1.1 and 37.1.2 in [10] and since (2.17) is the unique closed extension of $f$ to $\mathbf{R}^{M+1}$ we deduce

$$
\begin{aligned}
f(v, x) & =\sup _{u \in C} \inf _{y \in \operatorname{int} D}[\langle v, u\rangle+x y-g(u, y)], \\
& =\inf _{y \in D} \sup _{u \in \operatorname{int} C}[\langle v, u\rangle+x y-g(u, y)], \quad(v, x) \in \mathbf{R}^{M+1} .
\end{aligned}
$$

Noting that the continuous differentiability of $g$ on $(-\infty, 0)^{M} \times(0, \infty)$ is an immediate consequence of the existence and the uniqueness of the saddle points for (2.15), see [10], Theorem 35.8 and Corollary 37.5.3, we obtain that the result holds if

1. the interiors of the sets $C$ and $D$ are given by

$$
\begin{aligned}
& \operatorname{int} C=(-\infty, 0)^{M}, \\
& \text { int } D=(0, \infty) ;
\end{aligned}
$$


2. for $(u, y) \in(-\infty, 0)^{M} \times(0, \infty)$, the minimax values in $(2.18)$ are attained at a unique $(v, x) \in(0, \infty)^{M} \times \mathbf{R}$;

3. for $(v, x) \in(0, \infty)^{M} \times \mathbf{R}$, the minimax values in (2.19) are attained at a unique $(u, y) \in(-\infty, 0)^{M} \times(0, \infty)$.

For the set $C$ we have

$$
\begin{aligned}
C & \triangleq\left\{u \in \mathbf{R}^{M}: \bar{g}(u, y)<\infty \text { for all } y \in \mathbf{R}\right\} \\
& =\left\{u \in \mathbf{R}^{M}: \sup _{v \in \mathbf{R}_{+}^{M}}[\langle u, v\rangle-f(v, x)]<\infty \text { for some } x \in \mathbf{R}\right\} \\
& =\left\{u \in \mathbf{R}^{M}: \sup _{w \in \mathbf{S}^{M}}[\langle u, w\rangle-f(w, x)] \leq 0 \text { for some } x \in \mathbf{R}\right\},
\end{aligned}
$$

where at the last step we used (2.1). As $f \leq 0$ on $\mathbf{R}_{+}^{M} \times \mathbf{R}$, we have $C \subset(-\infty, 0]^{M}$. On the other hand, by (2.2) and (2.3), and, since, for every $w \in \mathbf{S}^{M}$, the function $f(w, \cdot)$ is increasing,

$$
\lim _{x \rightarrow \infty} \inf _{w \in \mathbf{S}^{M}} f(w, x)=0 .
$$

It follows that $(-\infty, 0)^{M} \subset C$, proving (2.20).

For the set $D$ we obtain

$$
\begin{aligned}
D & \triangleq\left\{y \in \mathbf{R}: \underline{g}(u, y)>-\infty \text { for all } u \in \mathbf{R}^{M}\right\} \\
& =\left\{y \in \mathbf{R}: \inf _{x \in \mathbf{R}}[x y-f(v, x)]>-\infty \text { for some } v \in \mathbf{R}^{M}\right\} .
\end{aligned}
$$

From (2.3) we deduce that $D \subset \mathbf{R}_{+}$. As $f \leq 0$ on $\mathbf{R}_{+}^{M} \times \mathbf{R}$, the point $y=0$ belongs to $D$. If $y>0$, then (2.1) implies the existence of $v \in(0, \infty)^{M}$ such that

$$
y=\frac{\partial f}{\partial x}(v, 1)
$$

and, therefore, for such $y$ and $v$,

$$
\inf _{x \in \mathbf{R}}[x y-f(v, x)]=y-f(v, 1)>-\infty
$$

Hence, $D=\mathbf{R}_{+}$, implying (2.21). 
Fix $v \in(0, \infty)^{M}$ and $x \in \mathbf{R}$. By the properties of $f$,

$$
\nabla f(v, x) \in(-\infty, 0)^{M} \times(0, \infty)=\operatorname{int} C \times \operatorname{int} D,
$$

implying that $(u, y) \triangleq \nabla f(v, x)$ is the unique saddle point of (2.19), see Corollary 37.5.3 in [10].

Fix now $u \in(-\infty, 0)^{M}$ and $y \in(0, \infty)$. As $f$ (viewed as a function on $\left.\mathbf{R}^{M+1}\right)$ is a closed saddle function and $(u, y)$ belongs to the interior of the effective domain of $g$, the minimax values in (2.18) are attained on a closed convex set of saddle points, namely, the subdifferential of $g$ evaluated at $(u, y)$, see Corollary 37.5.3 in [10]. To complete the proof it remains to be shown that this set is a singleton in $(0, \infty)^{M} \times \mathbf{R}$.

If $(\widehat{v}, \widehat{x})$ is a saddle point of $(2.18)$, then $(\widehat{v}, \widehat{x}) \in \operatorname{dom} f=\mathbf{R}_{+}^{M} \times \mathbf{R}$, and

$$
\begin{aligned}
g(u, y) & =\widehat{x} y+\langle\widehat{v}, u\rangle-f(\widehat{v}, \widehat{x})=\widehat{x} y+\sup _{v \in \mathbf{R}_{+}^{M}}[\langle v, u\rangle-f(v, \widehat{x})] \\
& =\langle\widehat{v}, u\rangle+\inf _{x \in \mathbf{R}}[x y-f(\widehat{v}, x)] .
\end{aligned}
$$

Accounting for the positive homogeneity property $(2.1)$ of $f(\cdot, \widehat{x})$ we deduce that

$$
\begin{aligned}
\widehat{x} y & =g(u, y), \\
\langle\widehat{v}, u\rangle-f(\widehat{v}, \widehat{x}) & =\sup _{v \in \mathbf{R}_{+}^{M}}[\langle v, u\rangle-f(v, \widehat{x})]=0, \\
\widehat{x} y-f(\widehat{v}, \widehat{x}) & =\inf _{x \in \mathbf{R}}[x y-f(\widehat{v}, x)] .
\end{aligned}
$$

The equality (2.22) defines $\widehat{x}$ uniquely. To show the uniqueness of $\widehat{v}$ we observe first that $\widehat{v} \in(0, \infty)^{M}$. Indeed, otherwise, we would have $f(\widehat{v}, x)=$ $0, x \in \mathbf{R}$, and the right side of (2.24) would be $-\infty$. Hence, $\widehat{v}$ can be decomposed as a product of $\widehat{w} \in \mathbf{S}^{M}$ and $\widehat{z}>0$. By (2.1) and (2.23),

$$
\langle\widehat{w}, u\rangle-f(\widehat{w}, \widehat{x})=\sup _{w \in \mathbf{S}^{M}}[\langle w, u\rangle-f(w, \widehat{x})]=0 .
$$

As $f(\cdot, \widehat{x})$ is strictly convex on $\mathbf{S}^{M}$, this identity determines $\widehat{w}$ uniquely. Finally, from (2.24) and the continuous differentiability of $f(\widehat{v}, \cdot)$ on $\mathbf{R}$ we deduce that

$$
y=\frac{\partial f}{\partial x}(\widehat{v}, \widehat{x})=\widehat{z} \frac{\partial f}{\partial x}(\widehat{w}, \widehat{x}),
$$

proving the uniqueness of $\widehat{z}$. 
Lemma 2.4. Let $f$ and $g$ be as in Lemma 2.3. Then $g$ satisfies the positive homogeneity property (2.10) and for $(v, x) \in(0, \infty)^{M} \times \mathbf{R}$ and $(u, y) \in$ $(-\infty, 0)^{M} \times(0, \infty)$ the relations below are equivalent:

1. Given $(u, y)$ the minimax values in (2.15) are attained at $(v, x)$.

2. Given $(v, x)$ the minimax values in (2.16) are attained at $(u, y)$.

3. We have $x=\frac{\partial g}{\partial y}(u, y)=g(u, 1)$ and $v=\frac{\partial g}{\partial u}(u, y)$.

4. We have $y=\frac{\partial f}{\partial x}(v, x)$ and $u=\frac{\partial f}{\partial v}(v, x)$.

Moreover, in this case, $f(v, x)=\langle u, v\rangle$ and $g(u, y)=x y$.

Proof. First, we observe that (2.10) for $g$ follows from the corresponding feature (2.1) for $f$ and the construction of $g$ in (2.15). The equivalence of items 1-4 follows from the characterization of saddle points in terms of the subdifferentials of conjugate functions, see Theorem 37.5 and Corollary 37.5.3 in [10]. The last assertion is straightforward.

Lemma 2.5. Let $f$ and $g$ be as in Lemma 2.3. Then $g \in \mathbf{G}^{1}$.

Proof. The continuous differentiability of $g=g(u, y)$ and the positive homogeneity condition (G4) have been already established in Lemmas 2.3 and 2.4, while (G3) (for $g$ not depending on $q$ ) follows trivially from (G2). Hence, $(\mathrm{G} 2)$ is the only remaining property to be proved. In view of (G4) it is sufficient to verify it only for $y=1$.

The function $g(\cdot, 1)$ is strictly increasing because its gradient is strictly positive in view of the characterization of saddle points to (2.15) by item 3 of Lemma 2.4. To show the strict convexity of $g(\cdot, 1)$, select $u_{1}$ and $u_{2}$, distinct elements of $(-\infty, 0)^{M}$, denote by $u_{3}$ their midpoint, and, for $i=1,2,3$, set $v_{i} \triangleq \frac{\partial g}{\partial u}\left(u_{i}, 1\right), x_{i} \triangleq g\left(u_{i}, 1\right)$. Since each $v_{i} \in(0, \infty)^{M}$, we can represent it as the product $v_{i}=z_{i} w_{i}$ of $z_{i} \in(0, \infty)$ and $w_{i} \in \mathbf{S}^{M}$. From Lemma 2.4 and the positive homogeneity property (2.1) we deduce that

$$
\begin{aligned}
\left\langle u_{i}, w_{i}\right\rangle-f\left(w_{i}, x_{i}\right) & =\sup _{w \in \mathbf{S}^{M}}\left[\left\langle u_{i}, w\right\rangle-f\left(w, x_{i}\right)\right]=0 \\
(y=) 1 & =z_{i} \frac{\partial f}{\partial x}\left(w_{i}, x_{i}\right)
\end{aligned}
$$


Since $\left(u_{i}\right)_{i=1,2,3}$ are distinct, so are $\left(v_{i}, x_{i}\right)_{i=1,2,3}$ (as $u_{i}=\frac{\partial f}{\partial v}\left(v_{i}, x_{i}\right)$ by the equivalence of items 3 and 4 in Lemma 2.4) and, hence, by (2.26), so are $\left(w_{i}, x_{i}\right)_{i=1,2,3}$. As $f(v, \cdot)$ is strictly concave on $\mathbf{R}$, we deduce from (2.25) that

$$
\begin{aligned}
f\left(w_{3}, x_{3}\right)= & \left\langle u_{3}, w_{3}\right\rangle=\frac{1}{2}\left(\left(\left\langle u_{1}, w_{3}\right\rangle-f\left(w_{3}, x_{1}\right)\right)+\left(\left\langle u_{2}, w_{3}\right\rangle-f\left(w_{3}, x_{2}\right)\right)\right) \\
& +\frac{1}{2}\left(f\left(w_{3}, x_{1}\right)+f\left(w_{3}, x_{2}\right)\right)<f\left(w_{3}, \frac{1}{2}\left(x_{1}+x_{2}\right)\right) .
\end{aligned}
$$

Since $f\left(w_{3}, \cdot\right)$ is strictly increasing on $\mathbf{R}$, we deduce that $x_{3}<\left(x_{1}+x_{2}\right) / 2$, implying the strict convexity of $g(\cdot, 1)$.

The assertion (2.5) of (G2)(a) follows from the monotonicity of $g(\cdot, 1)$ and the fact that, by Lemmas 2.3 and 2.4 , for every $x \in \mathbf{R}$ one can find $u \in(-\infty, 0)^{M}$ such that $x=g(u, 1)$.

For the proof of $(\mathrm{G} 2)(\mathrm{b})$ and $(\mathrm{G} 2)(\mathrm{c})$ we shall argue by contradiction. Let $\left(u_{n}\right)_{n>1}$ be a sequence in $(-\infty, 0)^{M}$ converging to a boundary point of $(-\infty, 0)^{M}$. Denote $x_{n} \triangleq g\left(u_{n}, 1\right), v_{n} \triangleq \frac{\partial g}{\partial u}\left(u_{n}, 1\right), n \geq 1$, and, contrary to (2.6), assume that the sequence $\left(v_{n}\right)_{n \geq 1}$ is bounded. Then, by the convexity of $g(\cdot, 1)$ and the boundedness of $\left(u_{n}\right)_{n \geq 1}$, the sequence $\left(x_{n}\right)_{n \geq 1}$ is also bounded. Hence, by passing to a subsequence, we can assume that the sequences $\left(v_{n}\right)_{n \geq 1}$ and $\left(x_{n}\right)_{n \geq 1}$ converge to finite limits $\widehat{v} \in \mathbf{R}_{+}^{M}$ and $\widehat{x} \in \mathbf{R}$, respectively. From Lemma 2.4 we deduce that

$$
u_{n}=\frac{\partial f}{\partial v}\left(v_{n}, x_{n}\right), \quad 1=\frac{\partial f}{\partial x}\left(v_{n}, x_{n}\right), \quad n \geq 1 .
$$

If $\widehat{v} \in(0, \infty)^{M}$, then

$$
\lim _{n \rightarrow \infty} u_{n}=\lim _{n \rightarrow \infty} \frac{\partial f}{\partial v}\left(v_{n}, x_{n}\right)=\frac{\partial f}{\partial v}(\widehat{v}, \widehat{x}) \in(-\infty, 0)^{M},
$$

contradicting our choice of $\left(u_{n}\right)_{n \geq 1}$. If, on the other hand, $\widehat{v} \in \partial \mathbf{R}_{+}^{M}$, then, by (2.1) and (2.2),

$$
\lim _{n \rightarrow \infty} f\left(v_{n}, x\right)=0, \quad x \in \mathbf{R} .
$$

Since the functions $f\left(v_{n}, \cdot\right)$ are concave, their pointwise convergence to 0 implies the convergence to 0 of its derivatives, uniformly on compact sets in $\mathbf{R}$, see Theorem 25.7 in [10]. It follows that

$$
\lim _{n \rightarrow \infty} \frac{\partial f}{\partial x}\left(v_{n}, x_{n}\right)=0
$$


contradicting the second equality in (2.27). This finishes the proof of $(\mathrm{G} 2)(\mathrm{b})$.

Let now $\left(u_{n}\right)_{n \geq 1}$ be a sequence in $(-\infty, 0)^{M}$ satisfying the conditions $(2.7)$ and (2.8) of $(\mathrm{G} 2)(\mathrm{c})$. Denote $x_{n} \triangleq g\left(u_{n}, 1\right)$ and, contrary to (2.9), assume that

$$
\limsup _{n \rightarrow \infty} g\left(u_{n}, 1\right)=\limsup _{n \rightarrow \infty} x_{n}>-\infty .
$$

As $g(\cdot, 1)$ is an increasing function on $(-\infty, 0)^{M},(2.7)$ implies the boundedness of the sequence $\left(x_{n}\right)_{n \geq 1}$ from above. Hence, by passing, if necessary, to a subsequence, we can assume that it converges to $\widehat{x} \in \mathbf{R}$. Define a sequence $\left(w_{n}\right)_{n \geq 1}$ in $\mathbf{S}^{M}$ by

$$
w_{n}=\frac{\partial g}{\partial u}\left(u_{n}, y_{n}\right)=y_{n} \frac{\partial g}{\partial u}\left(u_{n}, 1\right)
$$

for appropriate normalizing constants $y_{n}, n \geq 1$. By passing to a subsequence, we can assume that $\left(w_{n}\right)_{n \geq 1}$ converges to $\widehat{w}$ in the simplex $\operatorname{cl} \mathbf{S}^{M}$. From Lemma 2.4 we deduce that

$$
u_{n}=\frac{\partial f}{\partial v}\left(w_{n}, x_{n}\right), \quad n \geq 1
$$

If $\widehat{w} \in \mathbf{S}^{M}$, then

$$
\lim _{n \rightarrow \infty} u_{n}=\lim _{n \rightarrow \infty} \frac{\partial f}{\partial v}\left(w_{n}, x_{n}\right)=\frac{\partial f}{\partial v}(\widehat{w}, \widehat{x}) \in(-\infty, 0)^{M},
$$

contradicting (2.8). If $\widehat{w} \in \partial \mathbf{S}^{M}$, then, by (2.2), the sequence of concave functions $f\left(w_{n}, \cdot\right), n \geq 1$, on $\mathbf{R}$ converges to 0 pointwise and, therefore, also uniformly on compact sets. Accounting for Lemma 2.4 we deduce

$$
\lim _{n \rightarrow \infty}\left\langle u_{n}, w_{n}\right\rangle=\lim _{n \rightarrow \infty} f\left(w_{n}, x_{n}\right)=0,
$$

contradicting (2.7). This finishes the proof of $(\mathrm{G} 2)(\mathrm{c})$ and, with it, the proof of the lemma.

Lemma 2.6. Let $g=g(u, y):(-\infty, 0)^{M} \times(0, \infty) \rightarrow \mathbf{R}$ be in $\mathbf{G}^{1}$. Then there is a continuously differentiable function $f=f(v, x):(0, \infty)^{M} \times \mathbf{R} \rightarrow$ $(-\infty, 0)$ such that the minimax relations $(2.15)$ and $(2.16)$ hold and have unique saddle points. 
Proof. We follow similar arguments as in the proof of Lemma 2.3. To use the results of Section 37 in [10] we need to define the values of $g=g(u, y)$ at the boundary of the original domain by an appropriate closure operation. For $u \in(-\infty, 0)^{M}$ we set, by continuity, $g(u, 0) \triangleq 0$. Then for $y \geq 0$ we define, by lower semi-continuity,

$$
g(u, y) \triangleq \lim _{\varepsilon \rightarrow 0} \inf _{z \in B(u, \varepsilon)} g(z, y), \quad u \in \partial(-\infty, 0]^{M},
$$

where

$$
B(u, \varepsilon) \triangleq\left\{z \in(-\infty, 0)^{M}:|u-z| \leq \varepsilon\right\} .
$$

Note that in (2.28) the value of the limit may be infinite.

As in the proof of Lemma 2.3 we deduce the existence of a saddle function $f=f(v, x)$ defined on $(C \times \operatorname{int} D) \cup(D \times \operatorname{int} C)$, where

$$
\begin{aligned}
C & \triangleq\left\{v \in \mathbf{R}^{M}: f(v, x)<\infty \text { for all } x \in \mathbf{R}\right\} \\
& =\left\{v \in \mathbf{R}^{M}: \sup _{u \in(-\infty, 0]^{M}}[\langle v, u\rangle-g(u, y)]<\infty \text { for some } y \in \mathbf{R}_{+}\right\}, \\
D & \triangleq\left\{x \in \mathbf{R}: f(v, x)>-\infty \text { for all } v \in \mathbf{R}^{M}\right\} \\
& =\left\{x \in \mathbf{R}: \inf _{y \in \mathbf{R}_{+}}[x y-g(u, y)]>-\infty \text { for some } u \in(-\infty, 0]^{M}\right\},
\end{aligned}
$$

such that, for every $(v, x) \in(C \times \operatorname{int} D) \cup(D \times \operatorname{int} C)$,

$$
\begin{aligned}
f(v, x) & =\sup _{u \in(-\infty, 0]^{M}} \inf _{y \in \mathbf{R}_{+}}[\langle v, u\rangle+x y-g(u, y)], \\
& =\inf _{y \in \mathbf{R}_{+}} \sup _{u \in(-\infty, 0]^{M}}[\langle v, u\rangle+x y-g(u, y)],
\end{aligned}
$$

and, for every $(u, y) \in(-\infty, 0)^{M} \times(0, \infty)$,

$$
\begin{aligned}
g(u, y) & =\sup _{v \in C} \inf _{x \in \operatorname{int} D}[\langle v, u\rangle+x y-f(v, x)] \\
& =\inf _{x \in D} \sup _{v \in \operatorname{int} C}[\langle v, u\rangle+x y-f(v, x)] .
\end{aligned}
$$

As $g(u, y)=y g(u, 1)$, and, by $(\mathrm{G} 2)(\mathrm{c})$, for every $x \in \mathbf{R}$ there is $u \in$ $(-\infty, 0)^{M}$ such that $x \geq g(u, 1)$, we have

$$
D=\mathbf{R} \text {. }
$$


Choosing $y=0$ in the second description of $C$ above, we obtain

$$
\sup _{u \in(-\infty, 0]^{M}}[\langle u, v\rangle-g(u, 0)]=\sup _{u \in(-\infty, 0]^{M}}[\langle u, v\rangle]<\infty \text { iff } v \in \mathbf{R}_{+}^{M} .
$$

If $v \notin \mathbf{R}_{+}^{M}$, then there is $u_{0} \in(-\infty, 0)^{M}$ such that $\left\langle u_{0}, v\right\rangle>0$. By $(\mathrm{G} 2)(\mathrm{c})$, for every $y>0$,

$$
\lim _{n \rightarrow \infty} g\left(n u_{0}, y\right)=-\infty
$$

and, therefore,

$$
\sup _{u \in(-\infty, 0]^{M}}[\langle u, v\rangle-g(u, y)] \geq \limsup _{n \rightarrow \infty}\left[\left\langle n u_{0}, v\right\rangle-g\left(n u_{0}, y\right)\right]=\infty .
$$

It follows that

$$
C=\mathbf{R}_{+}^{M} .
$$

For $u \in(-\infty, 0)^{M}$ and $y>0$ we have $\nabla g(u, y) \in(0, \infty)^{M} \times \mathbf{R}$, implying that $(v, x) \triangleq \nabla g(u, y)$ is the unique saddle point of (2.30). In particular, we deduce that the minimax identities (2.15) and (2.30) have the same unique saddle points.

Let now $v \in(0, \infty)^{M}$ and $x \in \mathbf{R}$. As $(v, x)$ belongs to the interior of the effective domain of $f$, the minimax values in (2.29) are attained on a closed convex set of saddle points belonging to the subdifferential of $f$ evaluated at $(v, x)$, see Corollary 37.5.3 in [10]. We are going to show that this set is a singleton in $(-\infty, 0)^{M} \times(0, \infty)$.

Let $(\widehat{u}, \widehat{y})$ be a saddle point. Then

$$
(\widehat{u}, \widehat{y}) \in \operatorname{dom} g \subset(-\infty, 0]^{M} \times \mathbf{R}_{+},
$$

and

$$
\begin{aligned}
f(v, x) & =x \widehat{y}+\langle\widehat{u}, v\rangle-g(\widehat{u}, \widehat{y})=x \widehat{y}+\sup _{u \in(-\infty, 0]^{M}}[\langle u, v\rangle-g(u, \widehat{y})] \\
& =\langle\widehat{u}, v\rangle+\inf _{y \in \mathbf{R}_{+}}[x y-g(\widehat{u}, y)] .
\end{aligned}
$$

As $g(u, y)=y g(u, 1)$, we deduce that $\widehat{y}>0, \widehat{u} \neq 0$, and

$$
\begin{aligned}
f(v, x) & =\langle\widehat{u}, v\rangle \\
x & =g(\widehat{u}, 1) \\
\langle\widehat{u}, v\rangle-x \widehat{y} & =\sup _{u \in(-\infty, 0]^{M}}[\langle u, v\rangle-\widehat{y} g(u, 1)] .
\end{aligned}
$$


The attainability of the upper bound in (2.33) at $\widehat{u}$ implies that the subdifferential $\partial g(\widehat{u}, 1)$ is well-defined and $v \in \widehat{y} \partial g(\widehat{u}, 1)$. From $(\mathrm{G} 2)(\mathrm{b})$ we deduce that $g(\cdot, 1)$ is not subdifferentiable on the boundary of $(-\infty, 0]^{M}$ and, therefore, $\widehat{u} \in(-\infty, 0)^{M}$. As $g(\cdot, 1)$ is strictly convex on $(-\infty, 0)^{M},(2.32)$ defines $\widehat{u}$ uniquely, and, as $g(\cdot, 1)$ is differentiable on $(-\infty, 0)^{M}, \widehat{y}$ is uniquely determined by the equality

$$
v=\widehat{y} \frac{\partial g}{\partial u}(\widehat{u}, 1)
$$

The uniqueness of the saddle points $(\widehat{u}, \widehat{y})$ implies the continuous differentiability of $f$ on $(0, \infty)^{M} \times \mathbf{R}$. Finally, from (2.31) we deduce that $f<0$ on $(0, \infty)^{M} \times \mathbf{R}$.

Lemma 2.7. Let $g$ and $f$ be as in Lemma 2.6. Then $f$ satisfies the positive homogeneity condition (2.1) and, for $(v, x) \in(0, \infty)^{M} \times \mathbf{R}$ and $(u, y) \in$ $(-\infty, 0)^{M} \times(0, \infty)$, the assertions of Lemma 2.4 hold.

Proof. The positive homogeneity property (2.1) for $f$ is a consequence of the corresponding feature $(2.10)$ for $g$. The remaining assertions follow by the same arguments as in the proof of Lemma 2.4.

Lemma 2.8. Let $g$ and $f$ be as in Lemma 2.6. Then $f$ satisfies (F2).

Proof. Fix $x \in \mathbf{R}$. The positive homogeneity with respect to $v$ was already established in Lemma 2.7. By item 4 of Lemma 2.4, $\frac{\partial f}{\partial v}<0$, implying that the function $f(\cdot, x)$ is strictly decreasing.

Let $\left(w_{i}\right)_{i=1,2}$ be distinct points in $\mathbf{S}^{M}, w_{3}$ be their midpoint, and, for $i=1,2,3$, denote $u_{i} \triangleq \frac{\partial f}{\partial v}\left(w_{i}, x\right)$ and $y_{i} \triangleq \frac{\partial f}{\partial x}\left(w_{i}, x\right)$. By the characterizations of saddle points in Lemma 2.4, for $i=1,2,3$, we have

$$
f\left(w_{i}, x\right)=\left\langle u_{i}, w_{i}\right\rangle, \quad x=g\left(u_{i}, 1\right), \text { and } w_{i}=y_{i} \frac{\partial g}{\partial u}\left(u_{i}, 1\right) .
$$

From the last equality we deduce that the points $\left(u_{i}\right)_{i=1,2,3}$ are distinct. The uniqueness of saddle points for (2.16) then implies $\left\langle u_{3}, w_{i}\right\rangle<\left\langle u_{i}, w_{i}\right\rangle$, for $i=1,2$, and, therefore,

$$
\begin{aligned}
f\left(w_{3}, x\right) & =\left\langle u_{3}, w_{3}\right\rangle=\frac{1}{2}\left(\left\langle u_{3}, w_{1}\right\rangle+\left\langle u_{3}, w_{2}\right\rangle\right) \\
& <\frac{1}{2}\left(\left\langle u_{1}, w_{1}\right\rangle+\left\langle u_{2}, w_{2}\right\rangle\right)=\frac{1}{2}\left(f\left(w_{1}, x\right)+f\left(w_{2}, x\right)\right),
\end{aligned}
$$


proving the strict convexity of $f(\cdot, x)$ on $\mathbf{S}^{M}$.

Let now $\left(w_{n}\right)_{n \geq 1}$ be a sequence in $\mathbf{S}^{M}$ converging to $w \in \partial \mathbf{S}^{M}$. From (G2)(c) for every $\varepsilon>0$ we deduce the existence of $u(\varepsilon) \in(-\infty, 0)^{M}$ such that $g(u(\varepsilon), 1) \leq x$ and

$$
-\varepsilon \leq\langle u(\varepsilon), w\rangle=\lim _{n \rightarrow \infty}\left\langle u(\varepsilon), w_{n}\right\rangle .
$$

From the construction of $f$ in (2.16) we deduce $f(v, x) \geq\langle u(\varepsilon), v\rangle$ for every $v \in(0, \infty)^{M}$. It follows that

$$
\liminf _{n \rightarrow \infty} f\left(w_{n}, x\right) \geq \lim _{n \rightarrow \infty}\left\langle u(\varepsilon), w_{n}\right\rangle \geq-\varepsilon,
$$

proving (2.2).

Lemma 2.9. Let $g$ and $f$ be as in Lemma 2.6. Then $f$ satisfies (F4).

Proof. Fix $v \in(0, \infty)^{M}$. As, by item 4 of Lemma 2.4, $\frac{\partial f}{\partial x}>0$, the function $f(v, \cdot)$ is strictly increasing.

Let $\left(x_{i}\right)_{i=1,2}$ be distinct elements of $\mathbf{R}, x_{3} \triangleq \frac{1}{2}\left(x_{1}+x_{2}\right)$, and $u_{i} \triangleq \frac{\partial f}{\partial v}\left(v, x_{i}\right)$, $i=1,2,3$. From Lemma 2.7 we deduce

$$
g\left(u_{i}, 1\right)=x_{i}, \quad f\left(v, x_{i}\right)=\left\langle u_{i}, v\right\rangle, \quad i=1,2,3 .
$$

It follows that $\left(u_{i}\right)_{i=1,2,3}$ are distinct, and, hence, by the strict convexity of $g(\cdot, 1)$,

$$
g\left(\frac{1}{2}\left(u_{1}+u_{2}\right), 1\right)<\frac{1}{2}\left(g\left(u_{1}, 1\right)+g\left(u_{2}, 1\right)\right)=\frac{1}{2}\left(x_{1}+x_{2}\right)=x_{3} .
$$

From the uniqueness of saddle points in $(2.16)$ we deduce that if $g(u, 1)<x$ then $f(v, x)>\langle u, v\rangle$. It follows that

$$
f\left(v, x_{3}\right)>\left\langle\frac{1}{2}\left(u_{1}+u_{2}\right), v\right\rangle=\frac{1}{2}\left(f\left(v, x_{1}\right)+f\left(v, x_{2}\right)\right),
$$

proving the strict concavity of $f(v, \cdot)$.

For every $\varepsilon>0$ we can clearly find $u(\varepsilon) \in(-\infty, 0)^{M}$ such that $\langle u(\varepsilon), v\rangle \geq$ $-\varepsilon$. Denoting $x(\varepsilon) \triangleq g(u(\varepsilon), 1)$ we deduce

$$
\lim _{x \rightarrow \infty} f(v, x)>f(v, x(\varepsilon)) \geq\langle u(\varepsilon), v\rangle \geq-\varepsilon,
$$

proving (2.3). 
After these preparations we are ready to complete the proof of Theorem 2.2. From this moment, the functions $f$ and $g$ will depend on the "auxiliary" variable $q \in \mathbf{R}^{J}$.

Proof of Theorem 2.2. If $f=f(v, x, q) \in \mathbf{F}^{1}$, then, by Lemmas 2.3 and 2.5, the function

$$
g(u, y, q) \triangleq \sup _{v \in(0, \infty)^{M}} \inf _{x \in \mathbf{R}}[\langle v, u\rangle+x y-f(v, x, q)]
$$

satisfies (G2) and (G4) and is differentiable with respect to $u$ and $y$. Moreover, the concavity of $f(v, \cdot, \cdot)$ implies the convexity of $g(\cdot, y, \cdot)$. Conversely, if $g=g(u, y, q) \in \mathbf{G}^{1}$, then, by Lemmas 2.6-2.9, the function

$$
f(v, x, q) \triangleq \sup _{u \in(-\infty, 0)^{M}} \inf _{y \in(0, \infty)}[\langle u, v\rangle+x y-g(u, y, q)]
$$

satisfies (F2) and (F4) and is differentiable with respect to $v$ and $x$. Moreover, as $g$ is convex with respect to $(u, q), f$ is concave with respect to $(x, q)$.

The rest of the proof, namely, the equivalence of the differentiability of $f$ and $g$ with respect to $q$ and the relation (2.14), follows from the envelope theorem for saddle functions, Theorem A.1, given in Appendix A. Finally, we recall that for saddle functions the existence of derivatives implies the continuity of derivatives, see Theorem 35.8 and Corollary 35.7.1 in [10].

\subsection{Conjugacy relations between $\mathrm{F}^{2}$ and $\mathrm{G}^{2}$}

For $f \in \mathbf{F}^{2}$ and $g \in \mathbf{G}^{2}$, in addition to the matrices $A(f)$ and $B(g)$ given by (2.4) and (2.11), define the following matrices of second derivatives: for $m=1, \ldots, M$ and $i, j=1, \ldots, J$,

$$
\begin{gathered}
C^{m j}(f)(v, x, q) \triangleq \frac{v^{m}}{\frac{\partial f}{\partial x}}\left(\frac{\partial^{2} f}{\partial v^{m} \partial q^{j}}-\frac{1}{\frac{\partial^{2} f}{\partial x^{2}}} \frac{\partial^{2} f}{\partial v^{m} \partial x} \frac{\partial^{2} f}{\partial x \partial q^{j}}\right)(v, x, q), \\
D^{i j}(f)(v, x, q) \triangleq \frac{1}{\frac{\partial f}{\partial x}}\left(-\frac{\partial^{2} f}{\partial q^{i} \partial q^{j}}+\frac{1}{\frac{\partial^{2} f}{\partial x^{2}}} \frac{\partial^{2} f}{\partial x \partial q^{i}} \frac{\partial^{2} f}{\partial x \partial q^{j}}\right)(v, x, q),
\end{gathered}
$$

and

$$
\begin{aligned}
& E^{m j}(g)(u, y, q) \triangleq \frac{1}{\frac{\partial g}{\partial u^{m}}} \frac{\partial^{2} g}{\partial u^{m} \partial q^{j}}(u, y, q)=\frac{1}{\frac{\partial g}{\partial u^{m}}} \frac{\partial^{2} g}{\partial u^{m} \partial q^{j}}(u, 1, q), \\
& H^{i j}(g)(u, y, q) \triangleq \frac{1}{y} \frac{\partial^{2} g}{\partial q^{i} \partial q^{j}}(u, y, q)=\frac{\partial^{2} g}{\partial q^{i} \partial q^{j}}(u, 1, q)
\end{aligned}
$$


where in (2.36) and (2.37) we used the positive homogeneity (2.10) of $g$ with respect to $y$.

We use standard notations of linear algebra: for a square matrix $A$ of full rank, $A^{-1}$ denotes its inverse, and, for a matrix $B, B^{T}$ stands for its transpose.

Theorem 2.10. A function $f: \mathbf{A} \rightarrow(-\infty, 0)$ belongs to $\mathbf{F}^{2}$ if and only if it is conjugate to a function $g \in \mathbf{G}^{2}$ in the sense that (2.12) and (2.13) hold.

Moreover, if, for $q \in \mathbf{R}^{J}$, the vectors $a=(v, x, q) \in \mathbf{A}$ and $b=(u, y, q) \in$ $\mathbf{B}$ are conjugate in the sense of the equivalent conditions of items 1-4 of Theorem 2.2, then the matrices of the second derivatives for $f, A(f), C(f)$, and $D(f)$, defined in (2.4), (2.34), and (2.35), and the matrices of the second derivatives for $g, B(g), E(g)$, and $H(g)$, defined in (2.11), (2.36), and (2.37), are related by

$$
\begin{aligned}
& B(g)(b)=(A(f)(a))^{-1} \\
& E(g)(b)=-(A(f)(a))^{-1} C(f)(a), \\
& H(g)(b)=(C(f)(a))^{T}(A(f)(a))^{-1} C(f)(a)+D(f)(a) .
\end{aligned}
$$

Remark 2.11. Our choice of the specific form for the matrices $A(f)(a)$, $C(f)(a)$, and $D(f)(a)$ and $B(g)(b), E(g)(b)$, and $H(g)(b)$ was partially motivated by the fact that they are invariant under the transformations $(v, x, q) \rightarrow$ $(z v, x, q)$ and $(u, y, q) \rightarrow(u, z y, q), z>0$, which are natural in light of the positive homogeneity conditions (2.1) and (2.10).

\subsubsection{Proof of Theorem 2.10}

As in the proof of Theorem 2.2 we begin with several lemmas, where we omit the dependence on $q$.

Lemma 2.12. Let $f=f(v, x)$ and $g=g(u, y)$ be as in Lemma 2.3. Then the following assertions are equivalent:

1. $f$ is twice continuously differentiable and for all $v \in(0, \infty)^{M}$ and $x \in \mathbf{R}$ its Hessian matrix $K(v, x)=\left(K^{k l}(v, x)\right)_{k, l=1, \ldots, M+1}$ has full rank.

2. $g$ is twice continuously differentiable and for all $u \in(-\infty, 0)^{M}$ and $y \in(0, \infty)$ its Hessian matrix $L(u, y)=\left(L^{k l}(u, y)\right)_{k, l=1, \ldots, M+1}$ has full rank. 
Moreover, if $(v, x) \in(0, \infty)^{M} \times \mathbf{R}$ and $(u, y) \in(-\infty, 0)^{M} \times(0, \infty)$ are conjugate saddle points in the sense of Lemma 2.4, then $L(u, y)$ is inverse to $K(v, x)$.

Proof. The asserted equivalence is a well-known fact in the theory of saddle functions and is a direct consequence of the characterization of the gradients of the conjugate functions $f$ and $g$ given in Lemma 2.4 and the Implicit Function Theorem.

In the following statement we shall make the relationship between the Hessian matrices of $f$ and $g$ more explicit by taking into account the positive homogeneity property (2.10) of $g$.

Lemma 2.13. Let $f$ and $g$ be as in Lemma 2.3. Then the following assertions are equivalent:

1. $f$ is twice continuously differentiable and for all $v \in(0, \infty)^{M}$ and $x \in \mathbf{R}$

$$
\frac{\partial^{2} f}{\partial x^{2}}(v, x)<0
$$

and the Hessian matrix $K(v, x)$ of $f$ has full rank.

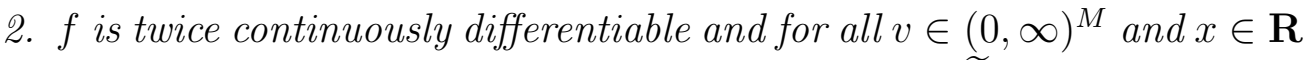
the inequality (2.41) holds and the $M \times M$ matrix $\widetilde{A}(v, x)$ with entries

$$
\widetilde{A}^{k l}(v, x) \triangleq\left(\frac{\partial^{2} f}{\partial v^{k} \partial v^{l}}-\frac{1}{\frac{\partial^{2} f}{\partial x^{2}}} \frac{\partial^{2} f}{\partial v^{k} \partial x} \frac{\partial^{2} f}{\partial v^{l} \partial x}\right)(v, x),
$$

has full rank.

3. $g$ is twice continuously differentiable and for all $u \in(-\infty, 0)^{M}$ and $y \in(0, \infty)$ the $M \times M$ matrix $\widetilde{B}(u, y)$ with entries

$$
\widetilde{B}^{k l}(u, y) \triangleq \frac{\partial^{2} g}{\partial u^{k} \partial u^{l}}(u, y)
$$

has full rank.

Moreover, if $(v, x) \in(0, \infty)^{M} \times \mathbf{R}$ and $(u, y) \in(-\infty, 0)^{M} \times(0, \infty)$ are conjugate saddle points in the sense of Lemma 2.4, then $\widetilde{B}(u, y)$ is the inverse of $\widetilde{A}(v, x)$. 
Proof. $1 \Longleftrightarrow 2$. From (2.41) and the construction of the matrix $\widetilde{A}(v, x)$ in (2.42) we deduce that for $a \in \mathbf{R}^{M}$ and $b \in \mathbf{R}$ the equation

$$
K(v, x)\left(\begin{array}{l}
a \\
b
\end{array}\right)=0
$$

is equivalent to

$$
b=-\frac{1}{\frac{\partial^{2} f}{\partial x^{2}}(v, x)} \sum_{m=1}^{M} \frac{\partial^{2} f}{\partial v^{m} \partial x}(v, x) a^{m}
$$

and

$$
\widetilde{A}(v, x) a=0 .
$$

It follows that under $(2.41)$ the matrices $K(v, x)$ and $\widetilde{A}(v, x)$ can have full rank only simultaneously.

$1 \Longleftrightarrow 3$. We fix arguments $(u, y)$ and $(v, x)$ satisfying the conjugacy relations of Lemma 2.4. From the definition of $\widetilde{B}=\widetilde{B}(u, y)$ in (2.43) we deduce that the Hessian matrix of $g$ at $(u, y)$ has the representation

$$
L(u, y)=\left(\begin{array}{cc}
\widetilde{B}(u, y) & \frac{\partial g}{\partial u}(u, 1) \\
\left(\frac{\partial g}{\partial u}(u, 1)\right)^{T} & 0
\end{array}\right)=\left(\begin{array}{cc}
\widetilde{B} & \frac{1}{y} v \\
\frac{1}{y} v^{T} & 0
\end{array}\right) .
$$

To simplify notations we shall also represent the Hessian matrix of $f$ at $(v, x)$ as

$$
K(v, x)=\left(\begin{array}{ll}
M & p \\
p^{T} & z
\end{array}\right)
$$

where $M$ is the Hessian matrix of $f(\cdot, x)$ at $v, p \triangleq\left(\frac{\partial^{2} f}{\partial v^{m} \partial x}(v, x)\right)_{m=1, \ldots, M}$ is the vector-column of mixed derivatives, and $z \triangleq \frac{\partial^{2} f}{\partial x^{2}}(v, x)$. Observe that the matrix $\widetilde{A}=\widetilde{A}(v, x)$ defined in (2.42) is given by

$$
\widetilde{A}=M-\frac{1}{z} p p^{T} \text {. }
$$

As $\widetilde{B}$ is a symmetric positive semi-definite matrix and $\frac{1}{y} v \neq 0$, the full rank of $\widetilde{B}$ implies the full rank of $L(u, y)$. Hence, by Lemma 2.12, under the conditions of either item 1 or item 3 , the Hessian matrices $K(v, x)$ and 
$L(u, y)$ have full rank and are inverse to each other. Denoting by $I$ the $M \times M$ identity matrix we deduce

$$
\begin{aligned}
\widetilde{B} M+\frac{1}{y} v p^{T} & =I, \\
\widetilde{B} p+\frac{1}{y} v z & =0 .
\end{aligned}
$$

If $z<0$, that is, (2.41) holds, then, by (2.44) and (2.45), $\widetilde{B} \widetilde{A}=I$. Hence, $\widetilde{B}$ is the inverse of $\widetilde{A}$ and, in particular, it has full rank, proving $1 \Longrightarrow 3$.

Conversely, if $\widetilde{B}$ has full rank, then $z=\frac{\partial^{2} f}{\partial x^{2}}(v, x) \neq 0$. Indeed, otherwise from the second equality in (2.45) we obtain $p=0$ contradicting the full rank of $K(v, x)$. Since, $f(v, \cdot)$ is concave, we deduce $z<0$, proving $3 \Longrightarrow 1$.

Proof of Theorem 2.10. If $(v, x, q) \in \mathbf{A}$ and $(u, y, q) \in \mathbf{B}$ satisfy the equivalent relations of items 1-4 of Theorem 2.2, then the matrices $A$ and $B$ defined in (2.4) and (2.11) and the matrices $\widetilde{A}$ and $\widetilde{B}$ defined in (2.42) and (2.43) are related by

$$
A^{k l}=\frac{v^{k} v^{l}}{y} \widetilde{A}^{k l}, \quad B^{k l}=\frac{y}{v^{k} v^{l}} \widetilde{B}^{k l}, \quad k, l=1, \ldots, M .
$$

Lemma 2.13 then implies (2.38) as well as the other assertions of the theorem except those involving the second derivatives with respect to $q$.

Assume first that $f \in \mathbf{F}^{2}$. We have to show that $g$ is two-times continuously differentiable and (2.39) and (2.40) hold. For $a \in \mathbf{R}^{M}$ define the function $h:(0, \infty)^{M} \times(-\infty, 0)^{M} \times \mathbf{R}^{J} \rightarrow \mathbf{R}^{M}$ by

$$
h(v, u, q) \triangleq\left(\frac{\partial f}{\partial v}(v, g(u, 1, q), q)-u\right)+a\left(\frac{\partial f}{\partial x}(v, g(u, 1, q), q)-1\right) .
$$

From Theorem 2.2 we deduce, for every $(u, y, q) \in \mathbf{B}$,

$$
h\left(\frac{\partial g}{\partial u}(u, y, q), u, q\right)=0
$$

Fix $\left(u_{0}, y_{0}, q_{0}\right) \in \mathbf{B}$, denote $v_{0} \triangleq \frac{\partial g}{\partial u}\left(u_{0}, y_{0}, q_{0}\right), x_{0} \triangleq g\left(u_{0}, 1, q_{0}\right)$, and choose

$$
a^{m} \triangleq-\left(\frac{\partial^{2} f}{\partial v^{m} \partial x} / \frac{\partial^{2} f}{\partial x^{2}}\right)\left(v_{0}, x_{0}, q_{0}\right), \quad m=1, \ldots, M .
$$


Direct computations show that, for $m, l=1, \ldots, M$ and $j=1, \ldots, J$,

$$
\begin{aligned}
& \frac{\partial h^{m}}{\partial v^{l}}\left(v_{0}, u_{0}, q_{0}\right)=\widetilde{A}^{m l}\left(v_{0}, x_{0}, q_{0}\right)=\frac{y_{0}}{v_{0}^{m} v_{0}^{l}} A^{m l}\left(v_{0}, x_{0}, q_{0}\right), \\
& \frac{\partial h^{m}}{\partial q^{j}}\left(v_{0}, u_{0}, q_{0}\right)=\frac{y_{0}}{v_{0}^{m}} C^{m j}\left(v_{0}, x_{0}, q_{0}\right) .
\end{aligned}
$$

By the Implicit Function Theorem the function $\frac{\partial g}{\partial u}=\frac{\partial g}{\partial u}(u, y, q)$ is continuously differentiable with respect to $q$ in a neighborhood of $\left(u_{0}, y_{0}, q_{0}\right)$ and the relation (2.39) holds at this point.

To prove the existence of the continuous second derivatives of $g$ with respect to $q$ and the remaining identity (2.40) we denote

$$
b^{j} \triangleq\left(\frac{\partial^{2} f}{\partial x \partial q^{j}} / \frac{\partial^{2} f}{\partial x^{2}}\right)\left(v_{0}, x_{0}, q_{0}\right), \quad j=1, \ldots, J .
$$

From Theorem 2.2 we deduce, for every $(u, y, q) \in \mathbf{B}$,

$$
\begin{aligned}
\frac{\partial g}{\partial q}(u, y, q)+b y= & -\frac{\partial f}{\partial q}\left(\frac{\partial g}{\partial u}(u, y, q), g(u, 1, q), q\right) \\
& +b \frac{\partial f}{\partial x}\left(\frac{\partial g}{\partial u}(u, y, q), g(u, 1, q), q\right)
\end{aligned}
$$

This implies the two-times continuous differentiability of $g$ with respect to $q$. Moreover, direct computations show that the differentiation of the above identity with respect to $q$ at $\left(u_{0}, y_{0}, q_{0}\right)$ yields $(2.40)$ at this point.

Assume now that $g \in \mathbf{G}^{2}$. To complete the proof we have to show that $f$ has continuous second derivatives involving $q$. By Theorem 2.2, for every $(v, x, q) \in \mathbf{A}$ we have the equalities

$$
\begin{array}{r}
\frac{\partial g}{\partial u}\left(\frac{\partial f}{\partial v}(v, x, q), \frac{\partial f}{\partial x}(v, x, q), q\right)-v=0, \\
\frac{\partial g}{\partial y}\left(\frac{\partial f}{\partial v}(v, x, q), \frac{\partial f}{\partial x}(v, x, q), q\right)-x=g\left(\frac{\partial f}{\partial v}(v, x, q), 1, q\right)-x=0, \\
\frac{\partial f}{\partial q}(v, x, q)+\frac{\partial g}{\partial q}\left(\frac{\partial f}{\partial v}(v, x, q), \frac{\partial f}{\partial x}(v, x, q), q\right)=0 .
\end{array}
$$

By Lemmas 2.12 and 2.13, the full rank of the matrix $B(u, y, q)$ implies the full rank of the Hessian matrix of $g(\cdot, \cdot, q)$ at $(u, y)$. An application of the Implicit Function Theorem to the first two equalities above then leads to the continuous differentiability of $\frac{\partial f}{\partial v}$ and $\frac{\partial f}{\partial x}$ with respect to $q$. By the third identity, this implies the existence and the continuity of $\frac{\partial^{2} f}{\partial q^{i} \partial q^{j}}$. 


\subsection{Stability under convergence}

Let $m$ be a non-negative integer and $U$ be an open subset of $\mathbf{R}^{d}$. Denote by $\mathbf{C}^{m}=\mathbf{C}^{m}\left(U, \mathbf{R}^{n}\right)$ the Fréchet space of $m$-times continuously differentiable maps $f: U \rightarrow \mathbf{R}^{n}$ with the topology generated by the semi-norms

$$
\|f\|_{m, C} \triangleq \sum_{0 \leq|k| \leq m} \sup _{x \in C}\left|D^{k} f(x)\right|,
$$

where $C$ is a compact subset of $U, k=\left(k_{1}, \ldots, k_{d}\right)$ is a multi-index of nonnegative integers, $|k| \triangleq \sum_{i=1}^{d} k_{i}$, and

$$
D^{k} \triangleq \frac{\partial^{|k|}}{\partial x_{1}^{k_{1}} \ldots \partial x_{d}^{k_{d}}} .
$$

In particular, for $m=0, D^{0}$ is the identity operator and $\|f\|_{0, C} \triangleq \sup _{x \in C}|f(x)|$.

The following results show that the conjugacy relations between the spaces $\mathbf{F}^{i}$ and $\mathbf{G}^{i}$ established in Theorems 2.2 and 2.10 remain stable under $\mathbf{C}^{i}$-convergence, $i=1,2$. These results will be used in the proofs of our main Theorems 4.1 and 4.2 to establish that the stochastic fields $F$ and $G$ have versions which are RCLL (right-continuous with left limits) in the time-variable $t$.

Theorem 2.14. Let $\left(f_{n}\right)_{n \geq 1}$ and $f$ belong to $\mathbf{F}^{1}$ and $\left(g_{n}\right)_{n \geq 1}$ and $g$ be their conjugate counterparts from $\mathbf{G}^{1}$. Then $\left(f_{n}\right)_{n \geq 1}$ converges to $f$ in $\mathbf{C}^{1}(\mathbf{A})$ if and only if $\left(g_{n}\right)_{n \geq 1}$ converges to $g$ in $\mathbf{C}^{1}(\mathbf{B})$.

Theorem 2.15. Let $\left(f_{n}\right)_{n \geq 1}$ and $f$ belong to $\mathbf{F}^{2}$ and $\left(g_{n}\right)_{n \geq 1}$ and $g$ be their conjugate counterparts from $\mathbf{G}^{2}$. Then $\left(f_{n}\right)_{n \geq 1}$ converges to $f$ in $\mathbf{C}^{2}(\mathbf{A})$ if and only if $\left(g_{n}\right)_{n \geq 1}$ converges to $g$ in $\mathbf{C}^{2}(\mathbf{B})$.

\subsubsection{Proofs of Theorems 2.14 and 2.15}

Proof of Theorem 2.14. Recall that for convex or saddle functions the convergence in $\mathbf{C}^{1}$ is equivalent to the pointwise convergence. We also remind the reader that the conjugacy operations, as in (2.12) and (2.13), are, in general, not continuous under this convergence and, hence, the result does not hold automatically. A standard verification method in this case is to show the equivalence of the pointwise convergence and the epi-convergence 
(or its analogs such as epi-hypo-convergence), under which the conjugacy operations are continuous; see Rockafellar and Wets [11], Theorem 11.34. We find it simpler to give a direct argument.

Assume first that $\left(f_{n}\right)_{n \geq 1}$ converges to $f$ in $\mathbf{C}^{1}(\mathbf{A})$. By the positive homogeneity condition (G4) and because they are saddle functions, it is sufficient to verify the pointwise convergence for $\left(g_{n}\right)_{n \geq 1}$ at $b=(u, y, q) \in \mathbf{B}$ with $y=1$. Fix $\varepsilon>0$ and find $u_{i} \in(-\infty, 0)^{M}, i=1,2$, such that $u_{1}<u<u_{2}$ and

$$
\left|g\left(b_{2}\right)-g\left(b_{1}\right)\right|<\varepsilon
$$

where $b_{i} \triangleq\left(u_{i}, 1, q\right)$. Denote, for $i=1,2$,

$$
a_{i}=\left(v_{i}, x_{i}, q\right) \triangleq\left(\frac{\partial g}{\partial u}\left(b_{i}\right), g\left(b_{i}\right), q\right)
$$

and, for $n \geq 1$,

$$
b_{i, n}=\left(u_{i, n}, 1, q\right) \triangleq\left(\frac{\partial f_{n}}{\partial v}\left(a_{i}\right), 1, q\right) .
$$

The conjugacy relations between $f_{n}$ and $g_{n}$ and between $f$ and $g$ imply that $g_{n}\left(b_{i, n}\right)=x_{i}$ and $u_{i}=\frac{\partial f}{\partial v}\left(a_{i}\right)$. From the $\mathbf{C}^{1}$-convergence of $\left(f_{n}\right)_{n \geq 1}$ to $f$ we deduce

$$
\lim _{n \rightarrow \infty} u_{i, n}=\lim _{n \rightarrow \infty} \frac{\partial f_{n}}{\partial v}\left(a_{i}\right)=\frac{\partial f}{\partial v}\left(a_{i}\right)=u_{i}, \quad i=1,2,
$$

and, hence, there is $n_{0}>1$ such that $u_{1, n}<u<u_{2, n}$ for $n \geq n_{0}$. Accounting for the monotonicity of the elements of $\mathbf{G}^{1}$ with respect to $u$ we obtain

$$
\begin{gathered}
g\left(b_{1}\right)<g(b)<g\left(b_{2}\right), \\
g\left(b_{1}\right)=g_{n}\left(b_{1, n}\right)<g_{n}(b)<g_{n}\left(b_{2, n}\right)=g\left(b_{2}\right), \quad n \geq n_{0},
\end{gathered}
$$

and then (2.47) yields

$$
\left|g_{n}(b)-g(b)\right|<\varepsilon, \quad n \geq n_{0},
$$

thus proving the pointwise, hence, also the $\mathbf{C}^{1}(\mathbf{B})$, convergence of $\left(g_{n}\right)_{n \geq 1}$ to $g$.

Assume now that $\left(g_{n}\right)_{n \geq 1}$ converges to $g$ in $\mathbf{C}^{1}(\mathbf{B})$. We follow the same path as in the proof of the previous implication. Fix $\varepsilon>0$, take $a=$ 
$(v, x, q) \in \mathbf{A}$ and let $a_{i}=\left(v_{i}, x_{i}, q\right) \in \mathbf{A}, i=1,2$, be such that $v_{1}>v>v_{2}$, $x_{1}<x<x_{2}$, and

$$
\left|f\left(a_{2}\right)-f\left(a_{1}\right)\right|<\varepsilon .
$$

Denote, for $i=1,2$,

$$
b_{i}=\left(u_{i}, y_{i}, q\right) \triangleq\left(\frac{\partial f}{\partial v}\left(a_{i}\right), \frac{\partial f}{\partial x}\left(a_{i}\right), q\right),
$$

and, for $n \geq 1$,

$$
a_{i, n}=\left(v_{i, n}, x_{i, n}, q\right) \triangleq\left(\frac{\partial g_{n}}{\partial u}\left(b_{i}\right), \frac{\partial g_{n}}{\partial y}\left(b_{i}\right), q\right) .
$$

From the conjugacy relations between $f_{n}$ and $g_{n}$ and between $f$ and $g$ we deduce that $f_{n}\left(a_{i, n}\right)=\left\langle u_{i}, v_{i, n}\right\rangle, f\left(a_{i}\right)=\left\langle u_{i}, v_{i}\right\rangle$, and $a_{i}=\left(\frac{\partial g}{\partial u}\left(b_{i}\right), \frac{\partial g}{\partial y}\left(b_{i}\right), q\right)$. As the $\mathbf{C}^{1}$-convergence of $\left(g_{n}\right)_{n \geq 1}$ to $g$ implies the convergence of $\left(a_{i, n}\right)_{n \geq 1}$ to $a_{i}$, there is $n_{0}>1$ such that, for $n \geq n_{0}, v_{1, n}>v>v_{2, n}, x_{1, n}<x<x_{2, n}$, and $\left|\left\langle u_{i}, v_{i, n}\right\rangle-\left\langle u_{i}, v_{i}\right\rangle\right|<\varepsilon$. Accounting for the monotonicity of the elements of $\mathbf{F}^{1}$ with respect to $v$ and $x$, we deduce

$$
\begin{gathered}
f\left(a_{1}\right)<f(a)<f\left(a_{2}\right), \\
f\left(a_{1}\right)-\varepsilon<f_{n}\left(a_{1, n}\right)<f_{n}(a)<f_{n}\left(a_{2, n}\right)<f\left(a_{2}\right)+\varepsilon, \quad n \geq n_{0},
\end{gathered}
$$

and, then, (2.48) implies

$$
\left|f_{n}(a)-f(a)\right|<2 \varepsilon, \quad n \geq n_{0},
$$

proving the pointwise (hence, also $\mathbf{C}^{1}(\mathbf{A})$ ) convergence of $\left(f_{n}\right)_{n \geq 1}$ to $f$.

For the proof of Theorem 2.15 we need some elementary identities for the matrices $A(f)$ and $C(f)$.

Lemma 2.16. For $f \in \mathbf{F}^{2}$, the matrix $A(f)$ defined in (2.4) satisfies

$$
\begin{aligned}
\sum_{m=1}^{M} A^{l m}(f) & =-v^{l} \frac{\partial^{2} f}{\partial v^{l} \partial x} / \frac{\partial^{2} f}{\partial x^{2}}, \quad l=1, \ldots, M, \\
\sum_{l, m=1}^{M} A^{l m}(f) & =-\frac{\partial f}{\partial x} / \frac{\partial^{2} f}{\partial x^{2}} .
\end{aligned}
$$


Proof. From the positive homogeneity condition (2.1) we deduce

$$
\begin{aligned}
& \sum_{m=1}^{M} v^{m} \frac{\partial^{2} f}{\partial v^{l} \partial v^{m}}=0 \\
& \sum_{m=1}^{M} v^{m} \frac{\partial^{2} f}{\partial x \partial v^{m}}=\frac{\partial f}{\partial x}
\end{aligned}
$$

and the result follows.

Lemma 2.17. For $f \in \mathbf{F}^{2}$, the matrix $C(f)$ defined in (2.34) satisfies

$$
\sum_{m=1}^{M} C^{m j}(f)=\frac{1}{\frac{\partial f}{\partial x}} \frac{\partial f}{\partial q^{j}}-\frac{1}{\frac{\partial^{2} f}{\partial x^{2}}} \frac{\partial^{2} f}{\partial q^{j} \partial x}, \quad j=1, \ldots, J
$$

Proof. The positive homogeneity property (2.1) yields Euler's identity:

$$
f=\sum_{m=1}^{M} v^{m} \frac{\partial f}{\partial v^{m}}
$$

which, in turn, implies

$$
\begin{aligned}
\frac{\partial f}{\partial x} & =\sum_{m=1}^{M} v^{m} \frac{\partial^{2} f}{\partial v^{m} \partial x}, \\
\frac{\partial f}{\partial q^{j}} & =\sum_{m=1}^{M} v^{m} \frac{\partial^{2} f}{\partial v^{m} \partial q^{j}}, \quad j=1, \ldots, J,
\end{aligned}
$$

proving (2.49).

Proof of Theorem 2.15. In view of Theorem 2.14, we only have to establish the uniform on compact sets convergences of second derivatives. Recall the notations $A(f), C(f)$, and $D(f)$, for the matrices defined in (2.4), (2.34) and (2.35), and $B(g), E(g)$, and $H(g)$, for the matrices defined in (2.11), (2.36), and (2.37).

Assume first that $\left(f_{n}\right)_{n \geq 1}$ converges to $f$ in $\mathbf{C}^{2}(\mathbf{A})$. Let $\left(b_{n}\right)_{n \geq 1}$ be a sequence in $\mathbf{B}$ that converges to $b \in \mathbf{B}$. By Theorem 2.14, the sequence $a_{n} \triangleq\left(\frac{\partial g_{n}}{\partial u}\left(b_{n}\right), \frac{\partial g_{n}}{\partial y}\left(b_{n}\right), q_{n}\right), n \geq 1$, converges to $a \triangleq\left(\frac{\partial g}{\partial u}(b), \frac{\partial g}{\partial y}(b), q\right)$. The convergence of $\left(f_{n}\right)_{n \geq 1}$ to $f$ in $\mathbf{C}^{2}(\mathbf{A})$ then implies the convergence of the 
matrices $\left(\left(A\left(f_{n}\right), C\left(f_{n}\right), D\left(f_{n}\right)\right)\left(a_{n}\right)\right)_{n \geq 1}$, to $(A(f), C(f), D(f))(a)$. By the identities (2.38), (2.39), and (2.40), this implies the convergence of the matrices $\left((B, E, H)\left(g_{n}\right)\left(b_{n}\right)\right)_{n \geq 1}$, to $(B, E, H)(g)(b)$, which, by the construction of these matrices, yields the convergence of all second derivatives of $g_{n}$ at $b_{n}, n \geq 1$, to the corresponding second derivatives of $g$ at $b$. This, clearly, implies the uniform on compact sets convergence of the second derivatives of $\left(g_{n}\right)_{n \geq 1}$ to $g$.

Similar arguments show that the $\mathbf{C}^{2}(\mathbf{B})$-convergence of $\left(g_{n}\right)_{n \geq 1}$ to $g$ implies that for every sequence $\left(a_{n}\right)_{n \geq 1}$ in $\mathbf{A}$ converging to $a \in \mathbf{A}$ the matrices $\left(\left(A\left(f_{n}\right), C\left(f_{n}\right), D\left(f_{n}\right)\right)\left(a_{n}\right)\right)_{n \geq 1}$ converge to $(A(f), C(f), D(f))(a)$. This, in turn, implies the convergence of the second derivatives of $f_{n}$ at $a_{n}, n \geq 1$, to the second derivatives of $f$ at $a$ if we account for the identity (2.49) for the matrix $C(f)$ and the equalities for the matrix $A(f)$ from Lemma 2.16.

\subsection{Additional conjugacy relations}

If $f \in \mathbf{F}^{1}$ and $g \in \mathbf{G}^{1}$ are conjugate in the sense that (2.12) and (2.13) hold true, then any extra condition for $f$ has its conjugate analog for $g$. Below we shall present several such extensions, which will appear in the description of the sample paths of the stochastic fields $F$ and $G$. Throughout this section we fix a constant $c>0$.

For a function $f: \mathbf{A} \rightarrow(-\infty, 0)$ define the following conditions:

(F6) If $M>1$, then for every $(x, q) \in \mathbf{R} \times \mathbf{R}^{J}$ and every sequence $\left(w_{n}\right)_{n \geq 1}$ in $\mathbf{S}^{M}$ converging to a boundary point of $\mathbf{S}^{M}$ we have

$$
\lim _{n \rightarrow \infty} \sum_{m=1}^{M} \frac{\partial f}{\partial v^{m}}\left(w_{n}, x, q\right)=-\infty .
$$

(F7) For every $a=(v, x, q) \in \mathbf{A}$ and $m=1, \ldots, M$,

$$
\frac{1}{c} \frac{\partial f}{\partial x}(a) \leq-v^{m} \frac{\partial f}{\partial v^{m}}(a) \leq c \frac{\partial f}{\partial x}(a) .
$$

(F8) For every $a \in \mathbf{A}$ and every $z \in \mathbf{R}^{M}$,

$$
\frac{1}{c}\langle z, z\rangle \leq\langle z, A(f)(a) z\rangle \leq c\langle z, z\rangle,
$$

where the matrix $A(f)(a)$ is defined in (2.4). 
(F9) For every $a=(v, x, q) \in \mathbf{A}$ and $m=1, \ldots, M$,

$$
-\frac{1}{c} \frac{\partial^{2} f}{\partial x^{2}}(a) \leq v^{m} \frac{\partial^{2} f}{\partial v^{m} \partial x}(a) \leq-c \frac{\partial^{2} f}{\partial x^{2}}(a) .
$$

For a function $g: \mathbf{B} \rightarrow \mathbf{R}$ define the following conditions:

(G6) For every $(y, q) \in(0, \infty) \times \mathbf{R}^{J}$ and every sequence $\left(u_{n}\right)_{n \geq 1}$ in $(-\infty, 0)^{M}$ converging to a boundary point of $(-\infty, 0)^{M}$ we have

$$
\lim _{n \rightarrow \infty} g\left(u_{n}, y, q\right)=\infty
$$

(G7) For every $(u, q) \in(-\infty, 0)^{M} \times \mathbf{R}^{J}$ and $m=1, \ldots, M$,

$$
\frac{1}{c} \leq-u^{m} \frac{\partial g}{\partial u^{m}}(u, 1, q) \leq c .
$$

(G8) For every $b \in \mathbf{B}$ and every $z \in \mathbf{R}^{M}$,

$$
\frac{1}{c}\langle z, z\rangle \leq\langle z, B(g)(b) z\rangle \leq c\langle z, z\rangle,
$$

where the matrix $B(g)(b)$ is defined in (2.11).

(G9) For every $(u, q) \in(-\infty, 0)^{M} \times \mathbf{R}^{J}$, the vector $z \in \mathbf{R}^{M}$ solving the linear equation:

$$
B(g)(u, 1, q) z=\mathbf{1},
$$

where $\mathbf{1} \triangleq(1, \ldots, 1) \in \mathbf{R}^{M}$, satisfies

$$
\frac{1}{c} \leq z^{m} \leq c, \quad m=1, \ldots, M .
$$

Note that for $g \in \mathbf{G}^{1}$ the condition (G6) implies (a) and (b) in (G2) and holds trivially when $M=1$ by (2.5).

Theorem 2.18. Let $f \in \mathbf{F}^{1}$ and $g \in \mathbf{G}^{1}$ be conjugate in the sense of (2.12) and (2.13). Then (F6) is equivalent to $(G 6)$ and $\left(F^{7}\right)$ is equivalent to $\left(G^{7}\right)$. If, in addition, $f \in \mathbf{F}^{2}$ and (hence) $g \in \mathbf{G}^{2}$ then (F8) is equivalent to (G8) and (F9) is equivalent to (G9). 


\subsubsection{Proof of Theorem 2.18}

The proof follows from the lemmas below, where $f \in \mathbf{F}^{1}$ and $g \in \mathbf{G}^{1}$ are conjugate as in (2.12).

Lemma 2.19. Suppose $M>1$. Then the conditions (F6) and (G6) are equivalent.

Proof. To simplify notations we shall omit the dependence of $f$ and $g$ on the irrelevant parameter $q$. Recall the notations $\partial A$ and $\mathrm{cl} A$ for the boundary and the closure of a set $A$.

$(\mathrm{F} 6) \Longrightarrow(\mathrm{G} 6)$. Let $\left(u_{n}\right)_{n \geq 1}$ be a sequence in $(-\infty, 0)^{M}$ converging to $\widehat{u} \in \partial(-\infty, 0)^{M}$. Denote $x_{n} \triangleq g\left(u_{n}, 1\right), n \geq 1$, and, contrary to (G6), suppose

$$
\liminf _{n \rightarrow \infty} g\left(u_{n}, 1\right)=\liminf _{n \rightarrow \infty} x_{n}<\infty .
$$

As $g(\cdot, 1)$ is an increasing function on $(-\infty, 0)^{M}$ and the sequence $\left(u_{n}\right)_{n \geq 1}$ is bounded from below, the sequence $\left(x_{n}\right)_{n \geq 1}$ is also bounded from below. Hence, by passing to a subsequence, we can assume that $\left(x_{n}\right)_{n \geq 1}$ converges to some $\widehat{x} \in \mathbf{R}$.

Denoting $v_{n} \triangleq \frac{\partial g}{\partial u}\left(u_{n}, 1\right)$ and $w_{n} \triangleq \frac{v_{n}}{\sum_{m=1}^{M} v_{n}^{m}}$ we deduce from items 3 and 4 of Theorem 2.2 and the positive homogeneity condition (2.1) for $f$ that

$$
u_{n}=\frac{\partial f}{\partial v}\left(v_{n}, x_{n}\right)=\frac{\partial f}{\partial v}\left(w_{n}, x_{n}\right), \quad n \geq 1 .
$$

As $w_{n} \in \mathbf{S}^{M}$, passing to a subsequence, we can assume that $\left(w_{n}\right)_{n \geq 1}$ converges to $\widehat{w} \in \operatorname{cl} \mathbf{S}^{M}$. If $\widehat{w} \in \mathbf{S}^{M}$, then

$$
\widehat{u}=\lim _{n \rightarrow \infty} u_{n}=\lim _{n \rightarrow \infty} \frac{\partial f}{\partial v}\left(w_{n}, x_{n}\right)=\frac{\partial f}{\partial v}(\widehat{w}, \widehat{x}) \in(-\infty, 0)^{M},
$$

contradicting our choice of $\widehat{u}$. If, on the other hand, $\widehat{w} \in \partial \mathbf{S}^{M}$, then, by (2.2) and the monotonicity of $f=f(v, x)$ with respect to $x$,

$$
\lim _{n \rightarrow \infty} f\left(w_{n}, x_{n}\right)=0 .
$$

It follows that, for every $v \in(0, \infty)^{M}$,

$$
\begin{aligned}
0 \geq f(v, \widehat{x}) & =\lim _{n \rightarrow \infty} f\left(v, x_{n}\right) \geq \lim _{n \rightarrow \infty}\left(f\left(w_{n}, x_{n}\right)+\left\langle\frac{\partial f}{\partial v}\left(w_{n}, x_{n}\right), v-w_{n}\right\rangle\right) \\
& =\lim _{n \rightarrow \infty}\left(f\left(w_{n}, x_{n}\right)+\left\langle u_{n}, v-w_{n}\right\rangle\right)=\langle\widehat{u}, v-\widehat{w}\rangle .
\end{aligned}
$$


Hence, if we extend, by continuity, the convex function $f(\cdot, \widehat{x})$ to the boundary of its domain by setting

$$
f(v, \widehat{x})=0, \quad v \in \partial(0, \infty)^{M},
$$

then its subdifferential $\partial f_{v}(\widehat{w}, \widehat{x})$ at $\widehat{w}$ contains $\widehat{u}$ and, therefore, is non-empty. In this case, there are $\widetilde{u} \in \partial f_{v}(\widehat{w}, \widehat{x})$ and a sequence $\left(\widetilde{v}_{n}\right)_{n \geq 1} \subset(0, \infty)^{M}$ convergent to $\widehat{w}$ such that

$$
\widetilde{u}=\lim _{n \rightarrow \infty} \frac{\partial f}{\partial v}\left(\widetilde{v}_{n}, \widehat{x}\right),
$$

see Theorem 25.6 in [10]. Denoting $\widetilde{w}_{n} \triangleq \frac{\widetilde{v}_{n}}{\sum_{m=1}^{M} \widetilde{v}_{n}^{m}}, n \geq 1$, and accounting for (2.1) we obtain

$$
\widetilde{u}=\lim _{n \rightarrow \infty} \frac{\partial f}{\partial v}\left(\widetilde{w}_{n}, \widehat{x}\right)
$$

which contradicts (F6).

(G6) $\Longrightarrow($ F6 $)$. Fix $x \in \mathbf{R}$, let $\left(w_{n}\right)_{n \geq 1}$ be a sequence in $\mathbf{S}^{M}$ converging to $w \in \partial \mathbf{S}^{M}$, and denote

$$
u_{n} \triangleq \frac{\partial f}{\partial v}\left(w_{n}, x\right), \quad y_{n} \triangleq \frac{\partial f}{\partial x}\left(w_{n}, x\right), \quad n \geq 1 .
$$

By (2.2), the concave functions $f\left(w_{n}, \cdot\right), n \geq 1$, converge to 0 . Hence, their derivatives also converge to 0 , implying that

$$
\lim _{n \rightarrow \infty} y_{n}=0 .
$$

Contrary to (F6) suppose $\left(u_{n}\right)_{n \geq 1}$ contains a bounded subsequence. Passing to a subsequence we can then assume that $\left(u_{n}\right)_{n \geq 1}$ converges to $u \in$ $(-\infty, 0]^{M}$.

By the equivalence of items 3 and 4 in Theorem 2.2,

$$
w_{n}=y_{n} \frac{\partial g}{\partial u}\left(u_{n}, 1\right), \quad x=g\left(u_{n}, 1\right), \quad n \geq 1 .
$$

In view of (2.50), the first equality implies that $u \notin(-\infty, 0)^{M}$. Hence, $u \in \partial(-\infty, 0)^{M}$, contradicting (G6) and the second equality.

Recall that a constant $c>0$ appearing in (F7)-(F9) and (G7)-(G9) is fixed. 
Lemma 2.20. The conditions $\left(F^{\prime 7}\right)$ and $(G 7)$ are equivalent.

Proof. Follows from the items 3 and 4 in the list of equivalent characterizations of saddle points in Theorem 2.2 and the positive homogeneity condition (2.10) for $g$.

From now on we assume, in addition, that $f \in \mathbf{F}^{2}$ and $g \in \mathbf{G}^{2}$.

Lemma 2.21. The conditions (F8) and (G8) are equivalent.

Proof. Follows from the inverse relation (2.38) between the matrices $A(f)(a)$ and $B(g)(b)$.

Lemma 2.22. The conditions (F9) and (G9) are equivalent.

Proof. By Lemma 2.16, the condition (F9) can be equivalently stated as

$$
\frac{1}{c} \leq(A(f)(a) \mathbf{1})^{m} \leq c, \quad m=1, \ldots, M,
$$

and the result follows from the inverse relation (2.38) between the matrices $A(f)(a)$ and $B(g)(b)$.

\subsection{The spaces $\widetilde{\mathbf{F}}^{1}, \widetilde{\mathbf{G}}^{1}$ and $\widetilde{\mathbf{F}}^{2}(c), \widetilde{\mathbf{G}}^{2}(c)$}

To simplify future references we define the following families of functions:

$$
\begin{array}{r}
\widetilde{\mathbf{F}}^{1} \triangleq\left\{f \in \mathbf{F}^{1}:(\mathrm{F} 6) \text { holds }\right\}, \\
\widetilde{\mathbf{G}}^{1} \triangleq\left\{g \in \mathbf{G}^{1}: \text { (G6) holds }\right\},
\end{array}
$$

and, for a constant $c>0$,

$$
\begin{array}{r}
\widetilde{\mathbf{F}}^{2}(c) \triangleq\left\{f \in \mathbf{F}^{2} \cap \widetilde{\mathbf{F}}^{1}:(\mathrm{F} 7)-(\mathrm{F} 9) \text { hold for given } c\right\}, \\
\widetilde{\mathbf{G}}^{2}(c) \triangleq\left\{g \in \mathbf{G}^{2} \cap \widetilde{\mathbf{G}}^{1}:(\mathrm{G} 7)-(\mathrm{G} 9) \text { hold for given } c\right\} .
\end{array}
$$

Note that when $M=1$ the conditions (F6) and (G6) hold trivially; in particular, $\widetilde{\mathbf{F}}^{1}=\mathbf{F}^{1}$ and $\widetilde{\mathbf{G}}^{1}=\mathbf{G}^{1}$.

From Theorems 2.2, 2.10, and 2.18 we immediately obtain

Theorem 2.23. A function $f: \mathbf{A} \rightarrow(-\infty, 0)$ belongs to $\widetilde{\mathbf{F}}^{1}$ if and only if it is conjugate to a function $g \in \widetilde{\mathbf{G}}^{1}$ in the sense of (2.12) and (2.13). Moreover, if $c>0$, then $f \in \widetilde{\mathbf{F}}^{2}(c)$ if and only if $g \in \widetilde{\mathbf{G}}^{2}(c)$. 


\section{Aggregate utility function}

Recall that the aggregate utility function $r=r(v, x)$ is given by

$$
r(v, x) \triangleq \sup _{x^{1}+\cdots+x^{M}=x} \sum_{m=1}^{M} v^{m} u_{m}\left(x^{m}\right), \quad v \in(0, \infty)^{M}, x \in \mathbf{R} .
$$

Theorems 3.1 and 3.2 below identify $r=r(v, x)$ as an element of $\widetilde{\mathbf{F}}^{1}$ and $\widetilde{\mathbf{F}}^{2}(c)$ under Assumptions 1.1 and 1.2, respectively. Note that throughout this section we interpret these families of functions, defined in Section 2.7, in the sense of Remark 2.1.

Theorem 3.1. Under Assumption 1.1 the function $r=r(v, x)$ belongs to $\widetilde{\mathbf{F}}^{1}$. Moreover, for every $(v, x) \in(0, \infty)^{M} \times \mathbf{R}$, the supremum in (3.1) is attained at the vector $\widehat{x} \in \mathbf{R}^{M}$ uniquely determined by (3.2) or, equivalently, (3.3) below:

$$
\begin{aligned}
v^{m} u_{m}^{\prime}\left(\widehat{x}^{m}\right) & =\frac{\partial r}{\partial x}(v, x), \\
u_{m}\left(\widehat{x}^{m}\right) & =\frac{\partial r}{\partial v^{m}}(v, x), \quad m=1, \ldots, M .
\end{aligned}
$$

Denote by $t_{m}=t_{m}(x)$ the risk-tolerance coefficients of the utility functions $u_{m}=u_{m}(x)$ :

$$
t_{m}(x) \triangleq-\frac{u_{m}^{\prime}(x)}{u_{m}^{\prime \prime}(x)}=\frac{1}{a_{m}(x)}, \quad x \in \mathbf{R}, m=1, \ldots, M .
$$

Hereafter, the symbol $\widehat{x}=\widehat{x}(v, x)$ is used to define the functional dependence of the maximal vector $\widehat{x}=\left(\widehat{x}^{m}\right)_{m=1, \ldots, M}$ from Theorem 3.1 on $v$ and $x$.

Theorem 3.2. Under Assumptions 1.1 and 1.2 the function $r=r(v, x)$ belongs to $\widetilde{\mathbf{F}}^{2}(c)$ with the same constant $c>0$ as in (1.3), the function $\widehat{x}=\widehat{x}(v, x)$ is continuously differentiable, and, for $l, m=1, \ldots, M$,

$$
\begin{aligned}
\frac{\partial \widehat{x}^{m}}{\partial x}(v, x) & =\frac{t_{m}\left(\widehat{x}^{m}\right)}{\sum_{k=1}^{M} t_{k}\left(\widehat{x}^{k}\right)}, \\
v^{l} \frac{\partial \widehat{x}^{m}}{\partial v^{l}}(v, x) & =v^{m} \frac{\partial \widehat{x}^{l}}{\partial v^{m}}(v, x)=t_{m}\left(\widehat{x}^{m}\right)\left(\delta_{l m}-\frac{t_{l}\left(\widehat{x}^{l}\right)}{\sum_{k=1}^{M} t_{k}\left(\widehat{x}^{k}\right)}\right),
\end{aligned}
$$


where $\delta_{l m} \triangleq 1_{\{l=m\}}$ is the Kronecker delta,

$$
\begin{aligned}
\frac{\partial^{2} r}{\partial x^{2}}(v, x) & =-\frac{\partial r}{\partial x}(v, x) \frac{1}{\sum_{k=1}^{M} t_{k}\left(\widehat{x}^{k}\right)}, \\
v^{m} \frac{\partial^{2} r}{\partial v^{m} \partial x}(v, x) & =\frac{\partial r}{\partial x}(v, x) \frac{t_{m}\left(\widehat{x}^{m}\right)}{\sum_{k=1}^{M} t_{k}\left(\widehat{x}^{k}\right)}, \\
v^{l} v^{m} \frac{\partial^{2} r}{\partial v^{l} \partial v^{m}}(v, x) & =\frac{\partial r}{\partial x}(v, x) t_{l}\left(\widehat{x}^{l}\right)\left(\delta_{l m}-\frac{t_{m}\left(\widehat{x}^{m}\right)}{\sum_{k=1}^{M} t_{k}\left(\widehat{x}^{k}\right)}\right),
\end{aligned}
$$

and, for the matrix $A(r)$ in (F5),

$$
\begin{aligned}
A^{l m}(r)(v, x) & \triangleq \frac{v^{l} v^{m}}{\frac{\partial r}{\partial x}}\left(\frac{\partial^{2} r}{\partial v^{l} \partial v^{m}}-\frac{1}{\frac{\partial^{2} r}{\partial x^{2}}} \frac{\partial^{2} r}{\partial v^{l} \partial x} \frac{\partial^{2} r}{\partial v^{m} \partial x}\right)(v, x) \\
& =t_{l}\left(\widehat{x}^{l}\right) \delta_{l m} .
\end{aligned}
$$

\subsection{Proofs of Theorems 3.1 and 3.2}

Proof of Theorem 3.1. Define the function $g=g(v, x, z):(0, \infty)^{M} \times \mathbf{R} \times$ $\mathbf{R}^{M-1} \rightarrow \mathbf{R}$ by

$$
g(v, x, z) \triangleq \sum_{m=1}^{M-1} v^{m} u_{m}\left(z^{m}\right)+v^{M} u_{M}\left(x-\sum_{m=1}^{M-1} z^{m}\right)
$$

and observe that

$$
r(v, x)=\sup _{z \in \mathbf{R}^{M-1}} g(v, x, z), \quad v \in(0, \infty)^{M}, x \in \mathbf{R} .
$$

For every $v \in(0, \infty)^{M}$, the function $g(v, \cdot, \cdot)$ is strictly concave, continuously differentiable, and, by (1.1) and (1.2), for every $x \in \mathbf{R}$,

$$
\lim _{|z| \rightarrow \infty} g(v, x, z)=-\infty
$$

It follows that the upper bound in (3.11) is attained at a unique $\widehat{z}=\widehat{z}(v, x)$ satisfying, for $m=1, \ldots, M-1$,

$$
0=\frac{\partial g}{\partial z^{m}}(v, x, \widehat{z})=v^{m} u_{m}^{\prime}\left(\widehat{z}^{m}\right)-v^{M} u_{M}^{\prime}\left(x-\sum_{k=1}^{M-1} \widehat{z}^{k}\right) .
$$


Hence, the upper bound in (3.1) is attained at the unique $\widehat{x}=\left(\widehat{x}^{m}\right)_{m=1, \ldots, M}$ given by

$$
\begin{aligned}
\widehat{x}^{m} & =\widehat{z}^{m}, \quad m=1, \ldots, M-1, \\
\widehat{x}^{M} & =x-\sum_{m=1}^{M-1} \widehat{z}^{m} .
\end{aligned}
$$

By Lemma A.3 in Appendix A the function $r(v, \cdot)$ is concave, differentiable, (hence, continuously differentiable), and

$$
\frac{\partial r}{\partial x}(v, x)=\frac{\partial g}{\partial x}(v, x, \widehat{z})=v^{M} u_{M}^{\prime}\left(x-\sum_{m=1}^{M-1} \widehat{z}^{k}\right)=v^{M} u_{M}^{\prime}\left(\widehat{x}^{M}\right),
$$

which jointly with (3.12) proves (3.2). As $u_{M}^{\prime}>0$ we have $\frac{\partial r}{\partial x}>0$ and, hence, $r(v, \cdot)$ is strictly increasing. From (1.1) we obtain

$$
\lim _{x \rightarrow \infty} r(v, x)=0 .
$$

Finally, the strict concavity of $r(v, \cdot)$ follows directly from the strict concavity of $\left(u_{m}\right)_{m=1, \ldots, M}$ and the attainability of the upper bound in (3.1), thus finishing the verification of (F4).

For $(x, z) \in \mathbf{R}^{M}$, the function $g(\cdot, x, z)$ is affine on $(0, \infty)^{M}$ and, in particular, convex and continuously differentiable. Hence, by Lemma A.4 in Appendix A, the function $r(\cdot, x)$ is convex, differentiable, (hence, continuously differentiable), and

$$
\frac{\partial r}{\partial v^{m}}(v, x)=\frac{\partial g}{\partial v^{m}}(v, x, \widehat{z})=u_{m}\left(\widehat{x}^{m}\right), \quad m=1, \ldots, M,
$$

proving (3.3). As $u_{m}<0$, the function $r(\cdot, x)$ is strictly decreasing. It is, clearly, positively homogeneous. Moreover, if $M>1$ then by (1.1)

$$
\lim _{n \rightarrow \infty} r\left(w_{n}, x\right)=0,
$$

for every sequence $\left(w_{n}\right)_{n \geq 1}$ in $\mathbf{S}^{M}$ converging to $w \in \partial \mathbf{S}^{M}$. Hence, to complete the verification of (F2) we only need to show the strict convexity of this function on $\mathbf{S}^{M}$.

Let $w_{1}$ and $w_{2}$ be distinct elements of $\mathbf{S}^{M}, w_{3}$ be their midpoint, and $\widehat{x}_{i}$ be the points in $\mathbf{R}^{M}$ where the upper bound in (3.1) is attained for $r\left(x, w_{i}\right)$, 
$i=1,2,3$. From (3.2) we deduce that the points $\left(\widehat{x}_{i}\right)_{i=1,2,3}$ are distinct and, hence,

$$
\begin{aligned}
r\left(w_{3}, x\right) & =\sum_{m=1}^{M} w_{3}^{m} u_{m}\left(\widehat{x}_{3}^{m}\right)=\frac{1}{2}\left(\sum_{m=1}^{M} w_{1}^{m} u_{m}\left(\widehat{x}_{3}^{m}\right)+\sum_{m=1}^{M} w_{2}^{m} u_{m}\left(\widehat{x}_{3}^{m}\right)\right) \\
& <\frac{1}{2}\left(\sum_{m=1}^{M} w_{1}^{m} u_{m}\left(\widehat{x}_{1}^{m}\right)+\sum_{m=1}^{M} w_{2}^{m} u_{m}\left(\widehat{x}_{2}^{m}\right)\right)=\frac{1}{2}\left(r\left(x, w_{1}\right)+r\left(x, w_{2}\right)\right) .
\end{aligned}
$$

This finishes the verification of (F2).

As we have already shown, $r=r(v, x)$ is a saddle function with welldefined partial derivatives at every point. In this case, $r$ is continuously differentiable, see Theorem 35.8 and Corollary 35.7.1 in [10], and, hence, satisfies (F1).

With (F3) following trivially from (F4), to complete the proof, we only have to verify (F6). Assume $M>1$ and let $\left(w_{n}\right)_{n \geq 1}$ be a sequence in $\mathbf{S}^{M}$ converging to $w \in \partial \mathbf{S}^{M}$. For $n \geq 1$ denote by $\widehat{x}_{n} \in \mathbf{R}^{M}$ the maximal allocation of $x$ corresponding to $w_{n}$. In view of $(3.13), \lim _{n \rightarrow \infty} \widehat{x}_{n}^{k}=\infty$ for every index $k$ with $w^{k}>0$. As $\sum_{m=1}^{M} \widehat{x}_{n}^{m}=x$, there is an index $m_{0}$ such that $\lim _{n \rightarrow \infty} \widehat{x}_{n}^{m_{0}}=-\infty$ and, therefore, accounting for (3.3) and (1.2),

$$
\lim _{n \rightarrow \infty} \sum_{m=1}^{M} \frac{\partial r}{\partial v^{m}}\left(w_{n}^{m}, x\right) \leq \lim _{n \rightarrow \infty} \frac{\partial r}{\partial v^{m_{0}}}\left(w_{n}^{m_{0}}, x\right)=\lim _{n \rightarrow \infty} u_{m_{0}}\left(\widehat{x}_{n}^{m_{0}}\right)=-\infty .
$$

Proof of Theorem 3.2. The proof relies on the Implicit Function Theorem. Define the function $h=h(v, x, y, z):(0, \infty)^{M} \times \mathbf{R} \times \mathbf{R}^{M} \times \mathbf{R} \rightarrow \mathbf{R}^{M+1}$ by

$$
\begin{aligned}
h^{m}(v, x, y, z) & =z-v^{m} u_{m}^{\prime}\left(y^{m}\right), \quad m=1, \ldots, M, \\
h^{M+1}(v, x, y, z) & =\sum_{m=1}^{M} y^{m}-x,
\end{aligned}
$$

and observe that, by Theorem 3.1,

$$
h\left(v, x, \widehat{x}(v, x), \frac{\partial r}{\partial x}(v, x)\right)=0, \quad(v, x) \in(0, \infty)^{M} \times \mathbf{R} .
$$


Fix $\left(v_{0}, x_{0}\right)$, set $y_{0} \triangleq \widehat{x}\left(v_{0}, x_{0}\right), z_{0} \triangleq \frac{\partial r}{\partial x}\left(v_{0}, x_{0}\right)$, and denote by $B=$ $\left(B^{k l}\right)_{k, l=1, \ldots, M+1}$ the Jacobian of $h\left(v_{0}, x_{0}, \cdot, \cdot\right)$ evaluated at $\left(y_{0}, z_{0}\right)$. Accounting for the fact that

$$
v_{0}^{m} u_{m}^{\prime}\left(y_{0}^{m}\right)=z_{0}, \quad m=1, \ldots, M
$$

we deduce

$$
\begin{aligned}
B^{k l} & =B^{l k}=-v_{0}^{k} u_{k}^{\prime \prime}\left(y_{0}^{k}\right) \delta_{k l}=\frac{z_{0}}{t_{k}\left(y_{0}^{k}\right)} \delta_{k l}, \quad k, l=1, \ldots, M, \\
B^{(M+1) m} & =B^{m(M+1)}=1, \quad m=1, \ldots, M, \\
B^{(M+1)(M+1)} & =0 .
\end{aligned}
$$

Direct computations show that the inverse matrix $C \triangleq B^{-1}$ is given by

$$
\begin{aligned}
C^{k l} & =C^{l k}=\frac{t_{k}\left(y_{0}^{k}\right)}{z_{0}}\left(\delta_{k l}-\frac{t_{l}\left(y_{0}^{l}\right)}{\sum_{i=1}^{M} t_{i}\left(y_{0}^{i}\right)}\right), \quad k, l=1, \ldots, M, \\
C^{(M+1) m} & =C^{m(M+1)}=\frac{t_{m}\left(y_{0}^{m}\right)}{\sum_{i=1}^{M} t_{i}\left(y_{0}^{i}\right)}, \quad m=1, \ldots, M, \\
C^{(M+1)(M+1)} & =-\frac{z_{0}}{\sum_{i=1}^{M} t_{i}\left(y_{0}^{i}\right)} .
\end{aligned}
$$

Since, for $m=1, \ldots, M+1$ and $l=1, \ldots, M$,

$$
\begin{aligned}
\frac{\partial h^{m}}{\partial x}\left(v_{0}, x_{0}, y_{0}, z_{0}\right) & =-\delta_{m(M+1)} \\
v^{l} \frac{\partial h^{m}}{\partial v^{l}}\left(v_{0}, x_{0}, y_{0}, z_{0}\right) & =-v^{m} u_{m}^{\prime}\left(y_{0}^{m}\right) \delta_{l m}=-z_{0} \delta_{l m},
\end{aligned}
$$

the Implicit Function Theorem implies the continuous differentiability of the functions $\widehat{x}=\widehat{x}(v, x)$ and $\frac{\partial r}{\partial x}=\frac{\partial r}{\partial x}(v, x)$ in the neighborhood of $\left(v_{0}, x_{0}\right)$ and 
the identities:

$$
\begin{aligned}
\frac{\partial \widehat{x}^{m}}{\partial x}\left(v_{0}, x_{0}\right) & =-\sum_{k=1}^{M+1} C^{m k} \frac{\partial h^{k}}{\partial x}\left(v_{0}, x_{0}, y_{0}, z_{0}\right)=C^{m(M+1)}, \\
v^{l} \frac{\partial \widehat{x}^{m}}{\partial v^{l}}\left(v_{0}, x_{0}\right) & =-\sum_{k=1}^{M+1} C^{m k} v^{l} \frac{\partial h^{k}}{\partial v^{l}}\left(v_{0}, x_{0}, y_{0}, z_{0}\right)=z_{0} C^{m l}, \\
\frac{\partial^{2} r}{\partial x^{2}}\left(v_{0}, x_{0}\right) & =-\sum_{k=1}^{M+1} C^{(M+1) k} \frac{\partial h^{k}}{\partial x}\left(v_{0}, x_{0}, y_{0}, z_{0}\right)=C^{(M+1)(M+1)}, \\
v^{l} \frac{\partial^{2} r}{\partial x \partial v^{l}}\left(v_{0}, x_{0}\right) & =-\sum_{k=1}^{M+1} C^{(M+1) k} v^{l} \frac{\partial h^{k}}{\partial v^{l}}\left(v_{0}, x_{0}, y_{0}, z_{0}\right)=z_{0} C^{(M+1) l}
\end{aligned}
$$

proving (3.5)-(3.6) and (3.7)-(3.8).

The continuous differentiability of $\frac{\partial r}{\partial v}=\frac{\partial r}{\partial v}(v, x)$ with respect to $v$ and the identity (3.9) follow from (3.3) and (3.6). Direct computations relying on (3.7), (3.8), and (3.9) lead to the expression (3.10) for $A(r)$, which jointly with (3.7) implies the validity of (F5) for $r=r(v, x)$.

Finally, accounting for (1.4) and observing that (1.3) can be equivalently stated as

$$
\frac{1}{c} \leq t_{m}(x) \leq c, \quad x \in \mathbf{R}, m=1, \ldots, M,
$$

we deduce that, for the function $r=r(v, x)$, the property (F7) follows from (3.2) and (3.3), (F8) is implied by (3.10), and (F9) follows from (3.7) and (3.8).

\section{Stochastic field of aggregate utilities and its conjugate}

We remind the reader of some terminology. For a set $A \subset \mathbf{R}^{d}$ a map $\xi: A \rightarrow$ $\mathbf{L}^{0}\left(\mathbf{R}^{n}\right)$ is called a random field; $\xi$ is continuous, convex, etc., if its sample paths $\xi(\omega): A \rightarrow \mathbf{R}^{n}$ are continuous, convex, etc., for all $\omega \in \Omega$. If $\xi$ and $\eta$ are random fields on $A$ then $\eta$ is a modification of $\xi$ if $\xi(x)=\eta(x)$ for every $x \in A$. A random field $X: A \times[0, T] \rightarrow \mathbf{L}^{0}\left(\mathbf{R}^{n}\right)$ is called a stochastic field if, for $t \in[0, T], X_{t} \triangleq X(\cdot, t): A \rightarrow \mathbf{L}^{0}\left(\mathscr{F}_{t}, \mathbf{R}^{n}\right)$, that is, the random variable $X_{t}$ is $\mathscr{F}_{t}$-measurable. 
Recall that the stochastic field of aggregate utilities is given by

$$
F_{t}(a) \triangleq \mathbb{E}\left[r(v, \Sigma(x, q)) \mid \mathscr{F}_{t}\right], \quad a=(v, x, q) \in \mathbf{A}, t \in[0, T]
$$

and its saddle conjugate with respect to $(v, x)$ is defined as

$$
G_{t}(b) \triangleq \sup _{v \in(0, \infty)^{M}} \inf _{x \in \mathbf{R}}\left[\langle v, u\rangle+x y-F_{t}(v, x, q)\right], \quad b=(u, y, q) \in \mathbf{B} .
$$

The sample paths of these stochastic fields are described in Theorems 4.1 and 4.2 which constitute the main results of the paper.

By $\mathbf{D}([0, T], \mathbf{X})$ we denote the space of RCLL (right-continuous with left limits) maps of $[0, T]$ into a metric space $\mathbf{X}$. Hereafter, for $i=1,2$, we view $\mathbf{F}^{i}$ as topological subspaces of the Fréchet spaces $\mathbf{C}^{i}(\mathbf{A})$ defined at the beginning of Section 2.5. A similar convention is also used for $\widetilde{\mathbf{F}}^{1}, \widetilde{\mathbf{G}}^{1}$ and $\widetilde{\mathbf{F}}^{2}(c), \widetilde{\mathbf{G}}^{2}(c)$.

Theorem 4.1. Suppose Assumption 1.1 and condition (1.5) hold. Then the stochastic fields $F=F_{t}(a)$ and $G=G_{t}(b)$ have modifications with sample paths in $\mathbf{D}\left([0, T], \widetilde{\mathbf{F}}^{1}\right)$ and $\mathbf{D}\left([0, T], \widetilde{\mathbf{G}}^{1}\right)$, respectively, and their left-limits $F_{t-}(\cdot)$ and $G_{t-}(\cdot)$ are conjugate to each other as in (4.1). Moreover, for every compact set $C \subset \mathbf{A}$

$$
\mathbb{E}\left[\left\|F_{T}(\cdot)\right\|_{1, C}\right]<\infty,
$$

and, for $a=(v, x, q) \in \mathbf{A}, t \in[0, T]$, and $i=1, \ldots, M+1+J$,

$$
\frac{\partial F_{t}}{\partial a^{i}}(a)=\mathbb{E}\left[\frac{\partial F_{T}}{\partial a^{i}}(a) \mid \mathscr{F}_{t}\right] .
$$

Theorem 4.2. Suppose Assumptions 1.1 and 1.2 and the condition (1.5) hold. Then the stochastic fields $F=F_{t}(a)$ and $G=G_{t}(b)$ have modifications with sample paths in $\mathbf{D}\left([0, T], \widetilde{\mathbf{F}}^{2}(c)\right)$ and $\mathbf{D}\left([0, T], \widetilde{\mathbf{G}}^{2}(c)\right)$, respectively, with the constant $c>0$ from Assumption 1.2. Moreover, for every compact set $C \subset \mathbf{A}$

$$
\mathbb{E}\left[\left\|F_{T}(\cdot)\right\|_{2, C}\right]<\infty,
$$

and, for $a=(v, x, q) \in \mathbf{A}, t \in[0, T]$, and $i, j=1, \ldots, M+1+J$,

$$
\frac{\partial^{2} F_{t}}{\partial a^{i} \partial a^{j}}(a)=\mathbb{E}\left[\frac{\partial^{2} F_{T}}{\partial a^{i} \partial a^{j}}(a) \mid \mathscr{F}_{t}\right] .
$$


Theorems 2.2, 2.10, and 2.18 in Section 2 allow us to establish various identities between the first and second derivatives of $F_{t}(\cdot)$ and $G_{t}(\cdot)$. We state one such corollary mentioned in Section 1 . Recall that $\mathbf{C}^{0}$ denotes the space of continuous functions with the topology of uniform convergence on compacts sets; see Section 2.5.

Corollary 4.3. Suppose Assumption 1.1 and the condition (1.5) hold. Then the stochastic fields

$$
U_{t}^{m}(a) \triangleq \frac{\partial F_{t}}{\partial v^{m}}(a), \quad a=(v, x, q) \in \mathbf{A}, m=1, \ldots, M
$$

have sample paths in $\mathbf{D}\left([0, T], \mathbf{C}^{0}(\mathbf{A})\right)$, the stochastic fields

$$
\begin{aligned}
X_{t}(u, q) & \triangleq G_{t}(b), \quad b=(u, 1, q) \in \mathbf{B}, \\
V_{t}^{m}(u, q) & \triangleq \frac{\partial G_{t}}{\partial u^{m}} \frac{1}{\sum_{l=1}^{M} \frac{\partial G_{t}}{\partial u^{l}}}(b), \quad b=(u, 1, q) \in \mathbf{B}, m=1, \ldots, M,
\end{aligned}
$$

have sample paths in $\mathbf{D}\left([0, T], \mathbf{C}^{0}\left((-\infty, 0)^{M}, \mathbf{R}^{J}\right)\right)$, and the following invertibility relations hold:

$$
\begin{aligned}
u^{m} & =U_{t}^{m}\left(V_{t}(u, q), X_{t}(u, q), q\right), m=1, \ldots, M, \\
x & =X_{t}\left(U_{t}(v, x, q), 1, q\right), \\
v^{m} & =V_{t}^{m}\left(U_{t}(v, x, q), 1, q\right), m=1, \ldots, M,
\end{aligned}
$$

where $u \in(-\infty, 0)^{M}, x \in \mathbf{R}, v \in \mathbf{S}^{M}, q \in \mathbf{R}^{J}$, and $t \in[0,1]$.

If, in addition, Assumption 1.2 holds then these stochastic fields have sample paths in $\mathbf{D}\left([0, T], \mathbf{C}^{1}\right)$.

Proof. The result follows directly from Theorems 4.1 and 4.2 and the conjugacy relations in items 3 and 4 of Theorem 2.2 as soon as we account for the positive homogeneity property (2.10) of the elements of $\mathbf{G}^{1}$.

Remark 4.4. Consider the price impact model from Section 1. Recall the definition of Pareto allocation $\pi(a)=\left(\pi^{m}(a)\right)$ from (1.8) and observe that by Theorems 3.1 and 4.1

$$
U_{t}^{m}(a)=\frac{\partial F_{t}}{\partial v^{m}}(a)=\mathbb{E}\left[u_{m}\left(\pi^{m}(a)\right) \mid \mathscr{F}_{t}\right] .
$$

Hence, $U_{t}^{m}(a)$ represents the expected utility of the $m$ th market maker at time $t$ given the Pareto allocation $\pi(a)$. By the invertibility relations in 
Corollary 4.3, the random variables $X_{t}(u, q)$ and $V_{t}(u, q)$ define the collective cash amount and the Pareto weights of the market makers at time $t$ when their current expected utilities are given by $u$ and they jointly own $q$ stocks.

\subsection{Proofs of Theorems 4.1 and 4.2}

For the proof of Theorem 4.1 we need the following result linking the condition (F6) in the definition of $\widetilde{\mathbf{F}}^{1}$ with the condition (C9) in Lemma C.3 from Appendix C.

Lemma 4.5. Let $M>1$. A function $f \in \mathbf{F}^{1}$ satisfies (F6) (that is, belongs to $\left.\widetilde{\mathbf{F}}^{1}\right)$ if and only if for every increasing sequence $\left(C_{n}\right)_{n \geq 1}$ of compact sets in $\mathbf{S}^{M}$ with $\cup_{n \geq 1} C_{n}=\mathbf{S}^{M}$ and for every compact set $D \subset \mathbf{R}^{1+J}$

$$
\lim _{n \rightarrow \infty} \sup _{w \in \mathbf{S}^{M} / C_{n}} \sup _{(x, q) \in D} \sum_{m=1}^{M} \frac{\partial f}{\partial v^{m}}(w, x, q)=-\infty .
$$

Proof. The "if" statement is straightforward. Hereafter we shall focus on the opposite implication.

To verify (4.6) we have to show that for $f \in \widetilde{\mathbf{F}}^{1}$ and every $a_{n}=\left(w_{n}, x_{n}, q_{n}\right) \in$ $\mathbf{S}^{M} \times \mathbf{R} \times \mathbf{R}^{J}, n \geq 1$, converging to $(w, x, q) \in \partial \mathbf{S}^{M} \times \mathbf{R} \times \mathbf{R}^{J}$ we have

$$
\lim _{n \rightarrow \infty} \sum_{m=1}^{M} \frac{\partial f}{\partial v^{m}}\left(a_{n}\right)=\lim _{n \rightarrow \infty}\left\langle\frac{\partial f}{\partial v}\left(a_{n}\right), \mathbf{1}\right\rangle=-\infty,
$$

where $\mathbf{1} \triangleq(1, \ldots, 1)$.

Let $\varepsilon>0$. Accounting for the convexity and the positive homogeneity of the functions $f\left(\cdot, x_{n}, q_{n}\right), n \geq 1$, on $(0, \infty)^{M}$ we deduce

$$
\begin{gathered}
\lim _{n \rightarrow \infty}\left\langle\frac{\partial f}{\partial v}\left(a_{n}\right), \mathbf{1}\right\rangle \leq \lim _{n \rightarrow \infty}\left\langle\frac{\partial f}{\partial v}\left(w_{n}+\varepsilon \mathbf{1}, x_{n}, q_{n}\right), \mathbf{1}\right\rangle \\
=\left\langle\frac{\partial f}{\partial v}(w+\varepsilon \mathbf{1}, x, q), \mathbf{1}\right\rangle=\left\langle\frac{\partial f}{\partial v}(w(\varepsilon), x, q), \mathbf{1}\right\rangle
\end{gathered}
$$

where $w(\varepsilon) \triangleq \frac{w+\varepsilon \mathbf{1}}{1+\varepsilon M}$ belongs to $\mathbf{S}^{M}$. By (F6), the passage to the limit when $\varepsilon \rightarrow 0$ yields (4.7). 
Proof of Theorem 4.1. As

$$
F_{T}(a)=r(v, \Sigma(x, q)), \quad a=(v, x, q) \in \mathbf{A},
$$

the assertions concerning the sample paths of $F_{T}=F_{T}(a)$ are immediate corollaries of the corresponding properties of $r=r(v, x)$ established in Theorem 3.1. By (1.5) we have that $F_{T}(a) \in \mathbf{L}^{1}, a \in \mathbf{A}$, and then, by Theorem B.1 from Appendix B, obtain (4.2). Lemma C.1 then implies that $F$ has sample paths in $\mathbf{D}\left([0, T], \mathbf{C}^{1}(\mathbf{A})\right)$ and that the equality (4.3) holds.

To verify that the sample paths of $F$ belong to $\mathbf{D}\left([0, T], \mathbf{F}^{1}\right)$ it is sufficient to match the properties (F1)-(F4) in the description of $\mathbf{F}^{1}$ with the properties (C1)-(C8) in Lemmas C.2 and C.3. For the most part these correspondences are straightforward with the links between (2.2) in (F2) or (2.3) in (F4) and their respective versions of $(\mathrm{C} 8)$ holding due to the equivalence of the pointwise and the uniform on compact sets convergences for a sequence of convex or saddle functions.

Note that in order to use (C8) in Lemma C.3 we still have to verify the integrability condition (C.6) in that lemma. Its adaption to (2.2) in (F2) takes the form:

$$
\mathbb{E}\left[\inf _{w \in \mathbf{S}^{M}} \inf _{(x, q) \in D} F_{T}(w, x, q)\right]>-\infty
$$

for every compact set $D \subset \mathbf{R}^{1+J}$, and follows because of (4.2) and since the sample paths of $F_{T}(\cdot)$ are decreasing with respect to $v$ (hence, $F_{T}(w, x, q)>$ $F_{T}(\mathbf{1}, x, q), w \in \mathbf{S}^{M}$, where $\left.\mathbf{1} \triangleq(1, \ldots, 1)\right)$. To perform a similar verification for the convergence (2.3) in (F4) we restrict the domain of $x$ to $[0, \infty)$. The analog of (C.6) then has the form:

$$
\mathbb{E}\left[\inf _{x \geq 0} \inf _{(v, q) \in D} F_{T}(v, x, q)\right]>-\infty,
$$

for every compact set $D \subset(0, \infty)^{M} \times \mathbf{R}^{J}$, and follows from (4.2) and the monotonicity of $F$ with respect to $x$. Thus, we have shown that the sample paths of $F$ belong to $\mathbf{D}\left([0, T], \mathbf{F}^{1}\right)$.

The connection between (C9) and (F6) has been established in Lemma 4.5. The adaptation of (C.6) to this case holds trivially as $\frac{\partial F}{\partial v}<0$. Hence, the sample paths of $F$ belong to $\mathbf{D}\left([0, T], \widetilde{\mathbf{F}}^{1}\right)$.

This finishes the proof of the assertions related to $F$. The remaining part, concerning $G$, follows directly from Theorems 2.23 and 2.14.

We divide the proof of Theorem 4.2 into lemmas. 
Lemma 4.6. Under the conditions of Theorem 4.2 the sample paths of the random field $F_{T}=F_{T}(\cdot)$ belong to the space $\widetilde{\mathbf{F}}^{2}(c)$ with the same constant $c>0$ as in Assumption 1.2. Moreover, (4.4) holds for every compact set $C \subset \mathbf{A}$.

Proof. The assertions for the sample paths of

$$
F_{T}(a)=r(v, \Sigma(x, q)), \quad a=(v, x, q) \in \mathbf{A},
$$

follow directly from the properties of $r=r(v, x)$ in Theorem 3.2.

To verify (4.4) fix a compact set $C \subset \mathbf{A}$. From the formulas for the second derivatives of $r=r(v, x)$ in Theorem 3.2 and from Assumption 1.2 we deduce the existence of a constant $b_{1}>0$ such that

$$
\left|\frac{\partial^{2} F_{T}}{\partial a^{i} \partial a^{j}}(a)\right| \leq b_{1} \frac{\partial F_{T}}{\partial x}(a)\left(1+|\psi|^{2}\right), \quad a \in C .
$$

From (3.7) we deduce that

$$
\frac{1}{c} \frac{\partial r}{\partial x}(v, x) \leq-M \frac{\partial^{2} r}{\partial x^{2}}(v, x) \leq c \frac{\partial r}{\partial x}(v, x), \quad x \in \mathbf{R},
$$

where $c>0$ is the constant from Assumption 1.2. This yields the exponential growth property

$$
e^{-y^{+} c / M+y^{-} /(c M)} \leq \frac{\frac{\partial r}{\partial x}(v, x+y)}{\frac{\partial r}{\partial x}(v, x)} \leq e^{-y^{+} /(c M)+y^{-} c / M}, \quad x, y \in \mathbf{R},
$$

where $x^{+} \triangleq \max (x, 0)$ and $x^{-} \triangleq(-x)^{+}$.

It follows that the right side of (4.8) is dominated by $b_{2}\left\|F_{T}\right\|_{1, D}$ for some constant $b_{2}>0$ and a compact set $D$ in $\mathbf{A}$ containing $C$. Hence, there is a constant $b_{3}>0$ such that

$$
\left\|F_{T}\right\|_{2, C} \leq b_{3}\left\|F_{T}\right\|_{1, D}
$$

and (4.4) holds because $\left\|F_{T}\right\|_{1, D}$ has a finite expected value by Theorem 4.1.

Lemma 4.7. Under the conditions of Theorem 4.2 the stochastic field $F=$ $F_{t}(a)$ has sample paths in $\mathbf{D}\left([0, T], \mathbf{C}^{2}(\mathbf{A})\right)$ and (4.5) holds. 
Proof. By Lemma 4.6, the random field $F_{T}(\cdot)$ has sample paths in $\mathbf{C}^{2}(\mathbf{A})$ and (4.4) holds. The result then follows from Lemma C.1.

Recall the notation $A(f)$ for the matrix defined in (2.4). A delicate point in the proof of Theorem 4.2 is to verify (F8) for the matrices $A\left(F_{t}\right), t \in[0, T]$.

For $a \in \mathbf{A}$, define the probability measure $\mathbb{R}(a)$ with

$$
\frac{d \mathbb{R}(a)}{d \mathbb{P}} \triangleq \frac{\partial^{2} F_{T}}{\partial x^{2}}(a) / \frac{\partial^{2} F_{0}}{\partial x^{2}}(a)
$$

the stochastic process

$$
R_{t}(a) \triangleq-\frac{\partial F_{t}}{\partial x}(a) / \frac{\partial^{2} F_{t}}{\partial x^{2}}(a), \quad t \in[0, T]
$$

and the random variables

$$
\tau^{m}(a) \triangleq t_{m}\left(\pi^{m}(a)\right), \quad m=1, \ldots, M,
$$

where $t_{m}=t_{m}(x)$ is the absolute risk-tolerance of $u_{m}=u_{m}(x)$ and $\pi^{m}(a)$ is a Pareto optimal allocation; see (3.4) and (1.8). Observe that $R(a)$ is a martingale under $\mathbb{R}(a)$, that, by (3.7),

$$
\sum_{m=1}^{M} \tau^{m}(a)=R_{T}(a),
$$

and, that by Assumption 1.2,

$$
\frac{1}{c} \leq \tau^{m} \leq c, \quad m=1, \ldots, M .
$$

Lemma 4.8. Assume that the conditions of Theorem 4.2 hold. Then the matrix $A\left(F_{t}\right)(a)$ is given by

$$
\begin{aligned}
A^{l m}\left(F_{t}\right)(a) & =\frac{1}{R_{t}(a)} \mathbb{E}_{\mathbb{R}(a)}\left[\tau^{l}(a)\left(\delta_{l m} \sum_{k=1}^{M} \tau^{k}(a)-\tau^{m}(a)\right) \mid \mathscr{F}_{t}\right] \\
& +\frac{1}{R_{t}(a)} \mathbb{E}_{\mathbb{R}(a)}\left[\tau^{l}(a) \mid \mathscr{F}_{t}\right] \mathbb{E}_{\mathbb{R}(a)}\left[\tau^{m}(a) \mid \mathscr{F}_{t}\right], \quad l, m=1, \ldots, M,
\end{aligned}
$$

where the probability measure $\mathbb{R}(a)$, the stochastic process $R(a)$, and the random variable $\tau(a)$ are defined in (4.9), (4.10), and (4.11), respectively and $\delta_{l m} \triangleq 1_{\{l=m\}}$ is the Kronecker delta. 
Moreover, for every $z \in \mathbf{R}^{n}$,

$$
\frac{1}{c}|z|^{2} \leq\left\langle z, A\left(F_{t}\right)(a) z\right\rangle \leq c|z|^{2},
$$

where the constant $c>0$ is given in Assumption 1.2.

Proof. From the expressions for the second derivatives of $r=r(v, x)$ in Theorem 3.2 we deduce

$$
\begin{aligned}
v^{m} \frac{\partial^{2} F_{T}}{\partial v^{m} \partial x}(a) & =-\frac{\partial^{2} F_{T}}{\partial x^{2}}(a) \tau^{m}(a), \\
v^{l} v^{m} \frac{\partial^{2} F_{T}}{\partial v^{l} \partial v^{m}}(a) & =-\frac{\partial^{2} F_{T}}{\partial x^{2}}(a) \tau^{l}(a)\left(\delta_{l m} \sum_{k=1}^{M} \tau^{k}(a)-\tau^{m}(a)\right),
\end{aligned}
$$

and the expression for $A\left(F_{t}\right)$ follows by direct computations.

To simplify notations, in the proof of (4.14), we omit the dependence on $a \in \mathbf{A}$ and consider the case $t=0$. Elementary calculations show that

$$
\begin{aligned}
\left\langle z, A\left(F_{0}\right) z\right\rangle & =\frac{1}{R_{0}}\left(\mathbb{E}_{\mathbb{R}}\left[R_{T} \sum_{m=1}^{M} \tau^{m} z_{m}^{2}-\langle\tau, z\rangle^{2}\right]+\left\langle\mathbb{E}_{\mathbb{R}}[\tau], z\right\rangle^{2}\right) \\
& =\frac{1}{R_{0}} \mathbb{E}_{\mathbb{R}}\left[R_{T} \sum_{m=1}^{M} \tau^{m} z_{m}^{2}-\left\langle\tau-\mathbb{E}_{\mathbb{R}}[\tau], z\right\rangle^{2}\right],
\end{aligned}
$$

where we used (4.12). This immediately implies the upper bound in (4.14):

$$
\left\langle z, A\left(F_{0}\right) z\right\rangle \leq \frac{1}{R_{0}} \mathbb{E}_{\mathbb{R}}\left[R_{T} \sum_{m=1}^{M} \tau^{m} z_{m}^{2}\right] \leq c|z|^{2},
$$

where we used the inequality (4.13) and the martingale property of $R$ under $\mathbb{R}$.

To verify the lower bound observe that by (4.13)

$$
\theta^{m} \triangleq \tau^{m}-\frac{1}{c} \geq 0 .
$$

We obtain

$$
\begin{aligned}
\left\langle z, A\left(F_{0}\right) z\right\rangle & =\frac{1}{R_{0}} \mathbb{E}_{\mathbb{R}}\left[R_{T} \sum_{m=1}^{M}\left(\frac{1}{c}+\theta^{m}\right) z_{m}^{2}-\left\langle\theta-\mathbb{E}_{\mathbb{R}}[\theta], z\right\rangle^{2}\right] \\
& =\frac{1}{c}|z|^{2}+\frac{1}{R_{0}} \mathbb{E}_{\mathbb{R}}\left[R_{T} \sum_{m=1}^{M} \theta^{m} z_{m}^{2}-\left\langle\theta-\mathbb{E}_{\mathbb{R}}[\theta], z\right\rangle^{2}\right] .
\end{aligned}
$$


As $R_{T}=\langle\tau, \mathbf{1}\rangle \geq\langle\theta, \mathbf{1}\rangle$, where $\mathbf{1} \triangleq(1, \ldots, 1)$, we deduce

$$
\begin{gathered}
R_{0}\left(\left\langle z, A\left(F_{0}\right) z\right\rangle-\frac{1}{c}|z|^{2}\right) \geq \mathbb{E}_{\mathbb{R}}\left[\langle\theta, \mathbf{1}\rangle \sum_{m=1}^{M} \theta^{m} z_{m}^{2}-\left\langle\theta-\mathbb{E}_{\mathbb{R}}[\theta], z\right\rangle^{2}\right] \\
=\mathbb{E}_{\mathbb{R}}\left[\frac{1}{\langle\theta, \mathbf{1}\rangle} \sum_{m=1}^{M} \theta^{m}\left(z_{m}\langle\theta, \mathbf{1}\rangle-\langle\theta, z\rangle\right)^{2}\right]+\left\langle\mathbb{E}_{\mathbb{R}}[\theta], z\right\rangle^{2} \geq 0 .
\end{gathered}
$$

Proof of Theorem 4.2. The inequality (4.4) and the fact that the sample paths of $F_{T}(\cdot)$ belong to $\widetilde{\mathbf{F}}^{2}(c)$ are established in Lemmas 4.6, while the identity (4.5) and the fact that the sample paths of $F=F_{t}(a)$ belong to $\mathbf{D}\left([0, T], \mathbf{C}^{2}(\mathbf{A})\right)$ are proved in Lemma 4.7. The remaining properties of $F$ follow from Lemma C.2 if we account for the properties of the sample paths for $F_{T}(\cdot)$ and use Lemma 4.8. Finally, the properties of the sample paths for $G=G_{t}(b)$ follow directly from those of $F$ and Theorems 2.23 and 2.15.

\section{A An envelope theorem for saddle functions}

In the proof of Theorem 2.2 we used the following version of the folklore "envelope" theorem for saddle functions. As usual, ri $C$ denotes the relative interior of a convex set $C$.

Theorem A.1. Let $C$ be a convex set in $\mathbf{R}^{n}$, $D$ be a convex set in $\mathbf{R}^{m}$, E be a convex open set in $\mathbf{R}^{l}, f=f(x, y, z): C \times D \times E \rightarrow \mathbf{R}$ be a function convex with respect to $x$ and concave with respect to $(y, z)$, and let $z_{0} \in E$. Denote

$$
g(z) \triangleq \sup _{y \in D} \inf _{x \in C} f(x, y, z), \quad z \in E,
$$

and assume that the maximin value $g\left(z_{0}\right)$ is attained at a unique $x_{0} \in \operatorname{ri} C$ and some (not necessarily unique) $y_{0} \in D$ and the function $f\left(x_{0}, y_{0}, \cdot\right)$ is differentiable at $z_{0}$.

Then the function $g: E \rightarrow \mathbf{R} \cup\{-\infty\}$ is concave, differentiable at $z_{0}$ (in particular, finite in a neighborhood of $z_{0}$ ) and

$$
\nabla g\left(z_{0}\right)=\frac{\partial f}{\partial z}\left(x_{0}, y_{0}, z_{0}\right) .
$$


Remark A.2. Theorem 5 in Milgrom and Segal [9] is the closest result to ours we could find in the literature. There, the convexity assumptions on $f$ are replaced by compactness requirements on $C$ and $D$.

The proof of Theorem A.1 relies on two lemmas of independent interest, which were used in the proof of Theorem 3.1. The first lemma is essentially known, see, for example, Corollary 3 in [9].

Lemma A.3. Let $f=f(x, y): \mathbf{R}^{n} \times \mathbf{R}^{m} \rightarrow \mathbf{R} \cup\{-\infty\}$ be a concave function and let $y_{0} \in \mathbf{R}^{m}$. Denote

$$
g(y) \triangleq \sup _{x \in \mathbf{R}^{n}} f(x, y), \quad y \in \mathbf{R}^{m},
$$

and assume that the upper bound $g\left(y_{0}\right)$ is attained at some (not necessarily unique) $x_{0} \in \mathbf{R}^{n}$ and the function $f\left(x_{0}, \cdot\right)$ is differentiable at $y_{0}$.

Then the function $g: \mathbf{R}^{m} \rightarrow \mathbf{R} \cup\{-\infty\}$ is concave, differentiable at $y_{0}$ and

$$
\nabla g\left(y_{0}\right)=\frac{\partial f}{\partial y}\left(x_{0}, y_{0}\right)
$$

Proof. The concavity of $g$ follows from the concavity of $f$ with respect to both arguments. As $g\left(y_{0}\right)=f\left(x_{0}, y_{0}\right)<\infty$, this concavity property implies that $g<\infty$. Since $g \geq f\left(x_{0}, \cdot\right)$, the function $g$ is finite in a neighborhood of $y_{0}$. It follows that $\partial g\left(y_{0}\right)$, the subdifferential of $g$ at $y_{0}$, is not empty.

If $y^{*} \in \partial g\left(y_{0}\right)$, then

$$
g(y) \leq g\left(y_{0}\right)+\left\langle y^{*}, y-y_{0}\right\rangle, \quad y \in \mathbf{R}^{m} .
$$

As $f\left(x_{0}, y\right) \leq g(y), y \in \mathbf{R}^{m}$, and $f\left(x_{0}, y_{0}\right)=g\left(y_{0}\right)$, it follows that

$$
f\left(x_{0}, y\right) \leq f\left(x_{0}, y_{0}\right)+\left\langle y^{*}, y-y_{0}\right\rangle, \quad y \in \mathbf{R}^{m} .
$$

Hence, $y^{*}$ belongs to the subdifferential of $f\left(x_{0}, \cdot\right)$ at $y_{0}$, and, therefore, $y^{*}=\frac{\partial f}{\partial y}\left(x_{0}, y_{0}\right)$. It follows that $y^{*}$ is the only element of $\partial g\left(y_{0}\right)$, proving the differentiability of $g$ at $y_{0}$ and the identity (A.1).

Lemma A.4. Let $C$ be a convex set in $\mathbf{R}^{n}$, $D$ be a convex open set in $\mathbf{R}^{m}$, $f=f(x, y): C \times D \rightarrow \mathbf{R}$ be a function concave with respect to $x$ and convex with respect to $y$, and let $y_{0} \in D$. Define the function

$$
g(y) \triangleq \sup _{x \in C} f(x, y), \quad y \in D
$$


and assume that the upper bound $g\left(y_{0}\right)$ is attained at a unique $x_{0} \in \operatorname{ri} C$ and the function $f\left(x_{0}, \cdot\right)$ is differentiable at $y_{0}$.

Then the function $g: D \rightarrow \mathbf{R} \cup\{\infty\}$ is convex, differentiable at $y_{0}$, and the identity (A.1) holds.

Remark A.5. The proof of Lemma A.4 will follow from the well-known analogous result in convex optimization, where the assumption of concavity in $x$ is replaced by the requirement that $C$ is compact, see, for example, Corollary 4.45 in Hiriart-Urruty and Lemaréchal [6].

Proof. The convexity of $g$ is straightforward. Let $\varepsilon>0$ be such that

$$
C(\varepsilon) \triangleq\left\{x \in C:\left|x-x_{0}\right| \leq \varepsilon\right\} \subset \operatorname{ri} C .
$$

If $\left(y_{n}\right)_{n \geq 1}$ is a sequence in $D$ converging to $y_{0}$, then the concave functions $f\left(\cdot, y_{n}\right), n \geq 1$, converge to $f\left(\cdot, y_{0}\right)$ uniformly on compact subsets of $C$. Since $x_{0}$ is the unique point of maximum for $f\left(\cdot, y_{0}\right)$, there is $n_{0}>0$ such that for every $n \geq n_{0}$ the concave function $f\left(\cdot, y_{n}\right)$ attains its maximum at some point $x_{n} \in C(\varepsilon)$. This argument implies the existence of $\delta>0$ such that

$$
g(y)=\sup _{x \in C(\varepsilon)} f(x, y)<\infty, \quad y \in D,\left|y-y_{0}\right|<\delta .
$$

As $C(\varepsilon)$ is a compact set, the result now follows from the well-known fact in convex optimization mentioned in Remark A.5.

Proof of Theorem A.1. The function $h=h(y, z): D \times E \rightarrow \mathbf{R} \cup\{-\infty\}$ given by

$$
h(y, z) \triangleq \inf _{x \in C} f(x, y, z), \quad y \in D, z \in E,
$$

is clearly concave. By Lemma A.4 the function $h\left(y_{0}, \cdot\right)$ is differentiable at $z_{0}$ and $\frac{\partial h}{\partial z}\left(y_{0}, z_{0}\right)=\frac{\partial f}{\partial z}\left(x_{0}, y_{0}, z_{0}\right)$. An application of Lemma A.3 completes the proof.

\section{B Integrability of saddle random fields}

The following theorem states that the pointwise integrability of a saddle random field $\xi$ implies the integrability of pseudo-norms $\|\xi\|_{0, C}$ for compact sets $C$. It also implies the integrability of $\|\xi\|_{1, C}$ if, in addition, the sample 
paths of $\xi$ are differentiable. See Section 2.5 for the definition of the seminorm $\|\cdot\|_{m, C}$. This result is used in the proof of Theorem 4.1.

We fix a probability space $(\Omega, \mathscr{F}, \mathbb{P})$ and denote $\mathbf{L}^{1}=\mathbf{L}^{1}(\Omega, \mathscr{F}, \mathbb{P})$ the Banach space of integrable random variables.

Theorem B.1. Let $U \subset \mathbf{R}^{n}$ and $V \subset \mathbf{R}^{m}$ be open sets and $\xi: U \times V \rightarrow \mathbf{L}^{1}$ be a random field with sample paths in the space of concave-convex functions on $U \times V$. Then for every compact set $C \subset U \times V$

$$
\mathbb{E}\left[\|\xi\|_{0, C}\right]<\infty .
$$

If, in addition, the sample paths of $\xi$ belong to $\mathbf{C}^{1}(U)$, then

$$
\mathbb{E}\left[\|\xi\|_{1, C}\right]<\infty .
$$

The proof is divided into lemmas.

Lemma B.2. Let $U$ be an open set in $\mathbf{R}^{d}, f: U \rightarrow \mathbf{R}$ be a convex function, $C$ be a compact subset of $U$, and $\varepsilon>0$ be such that

$$
C(\varepsilon) \triangleq\left\{x \in \mathbf{R}^{d}: \inf _{y \in C}|x-y| \leq \varepsilon\right\} \subset U
$$

Then for every $y \in C$ we have

$$
\min _{x \in C} f(x) \geq f(y)+\frac{\sup _{x \in C}|x-y|}{\varepsilon}\left(f(y)-\max _{x \in C(\varepsilon)} f(x)\right) .
$$

Proof. Fix $y \in C$. For every $x \in C$ there is $z \in \partial C(\varepsilon)$ such that $y$ is a convex combination of $x$ and $z: y=t x+(1-t) z$ for some $t \in(0,1)$. Using the fact that $|y-z| \geq \varepsilon$ we obtain

$$
\frac{1-t}{t}=\frac{|x-y|}{|y-z|} \leq \frac{\sup _{x \in C}|x-y|}{\varepsilon} .
$$

The convexity of $f$ implies

$$
f(y) \leq t f(x)+(1-t) f(z),
$$

or, equivalently,

$$
f(x) \geq f(y)+\frac{1-t}{t}(f(y)-f(z)),
$$

which, in view of (B.5), yields (B.4). 
Lemma B.3. In addition to the conditions of Lemma B.2 assume that $f \in$ $\mathbf{C}^{1}(U)$. Then

$$
\|f\|_{1, C} \leq\left(\frac{2 \sqrt{d}}{\varepsilon}+1\right)\|f\|_{0, C(\varepsilon)} .
$$

Proof. For $y \in C$ and $x \in C(\varepsilon)$ we obtain from the convexity of $f$ :

$$
f(x)-f(y) \geq\langle x-y, \nabla f(y)\rangle .
$$

It follows that

$$
2\|f\|_{0, C(\varepsilon)} \geq \sup _{y \in C} \sup _{\{x:|x-y| \leq \varepsilon\}}(\langle x-y, \nabla f(y)\rangle)=\varepsilon \sup _{y \in C}|\nabla f(y)| .
$$

Since,

$$
|\nabla f(y)| \triangleq \sqrt{\sum_{i=1}^{d}\left(\frac{\partial f}{\partial x_{i}}(y)\right)^{2}} \geq \frac{1}{\sqrt{d}} \sum_{i=1}^{d}\left|\frac{\partial f}{\partial x_{i}}(y)\right|,
$$

we obtain

$$
|f(y)|+\sum_{i=1}^{d}\left|\frac{\partial f}{\partial x_{i}}(y)\right| \leq\left(\frac{2 \sqrt{d}}{\varepsilon}+1\right)\|f\|_{0, C(\varepsilon)},
$$

which clearly implies (B.6).

Lemma B.4. Let $U$ be an open set in $\mathbf{R}^{d}, \xi=\xi(x): U \rightarrow \mathbf{L}^{1}$ be a random field with sample paths in the space of convex functions on $U$. Then, for every compact set $C \subset U$,

$$
\mathbb{E}\left[\|\xi\|_{0, C}\right]<\infty .
$$

If, in addition, the sample paths of $\xi$ belong to $\mathbf{C}^{1}(U)$ then

$$
\mathbb{E}\left[\|\xi\|_{1, C}\right]<\infty .
$$

Proof. Let us first show that for every compact set $C \subset U$

$$
\mathbb{E}\left[\max _{x \in C} \xi(x)\right]<\infty .
$$

Without restricting generality we can assume that $C$ is the closed convex hull of a finite family $\left(x_{i}\right)_{i=1, \ldots, I}$ in $U$. From the convexity of $\xi$ we then deduce

$$
\max _{x \in C} \xi(x)=\max _{i=1, \ldots, I} \xi\left(x_{i}\right),
$$


and (B.9) follows from the assumption $\xi(x) \in \mathbf{L}^{1}, x \in U$.

Since $C$ is a compact set in $U$, for sufficiently small $\varepsilon>0$ the set $C(\varepsilon)$ defined in (B.3) is also a compact subset of $U$. By (B.9) and Lemma B.2 we obtain

$$
\mathbb{E}\left[\min _{x \in C} \xi(x)\right]>-\infty
$$

which implies (B.7).

Finally, if $f \in \mathbf{C}^{1}$, then (B.8) follows from (B.7) and Lemma B.3.

Proof of Theorem B.1. It is sufficient to consider the case $C=C_{1} \times C_{2}$, where $C_{1}$ and $C_{2}$ are compact subsets of $U$ and $V$, respectively. To prove (B.1) it is enough to show that

$$
\sup _{x \in C_{1}} \sup _{y \in C_{2}} \xi(x, y) \in \mathbf{L}^{1} .
$$

Indeed, having established (B.10) for every random field $\xi$ and every pair of open sets $U$ and $V$ satisfying the conditions of the lemma we deduce

$$
\inf _{x \in C_{1}} \inf _{y \in C_{2}} \xi(x, y)=-\sup _{x \in C_{1}} \sup _{y \in C_{2}}(-\xi(x, y)) \in \mathbf{L}^{1},
$$

which, jointly with (B.10), implies (B.1). To verify (B.10) observe that the random field

$$
\eta(y) \triangleq \sup _{x \in C_{1}} \xi(x, y), \quad y \in V
$$

has sample paths in the space of convex functions and, by Lemma B.4, $\eta(y) \in$ $\mathbf{L}^{1}$. Another application of Lemma B.4 yields $\|\eta\|_{0, C_{2}} \in \mathbf{L}^{1}$, which clearly implies (B.10).

To verify (B.2), choose $\varepsilon>0$ so that the sets $C_{1}(\varepsilon)$ and $C_{2}(\varepsilon)$ defined by (B.3) are still in $U$ and $V$. Then, by Lemma B.3, there is $c=c(\varepsilon)>0$ such that for every $x \in C_{1}$ and $y \in C_{2}$

$$
\begin{aligned}
\|\xi(x, \cdot)\|_{1, C_{2}}+\|\xi(\cdot, y)\|_{1, C_{1}} & \leq c\left(\|\xi(x, \cdot)\|_{0, C_{2}(\varepsilon)}+\|\xi(\cdot, y)\|_{0, C_{1}(\varepsilon)}\right) \\
& \leq 2 c\|\xi\|_{0, C_{1}(\varepsilon) \times C_{2}(\varepsilon)}
\end{aligned}
$$

and the result follows. 


\section{Stochastic fields of martingales}

This appendix contains the results concerning the sample paths of stochastic fields of martingales used in the proofs of Theorems 4.1 and 4.2.

We fix a complete filtered probability space $\left(\Omega, \mathscr{F},\left(\mathscr{F}_{t}\right)_{t \in[0, T]}, \mathbb{P}\right)$ with $\mathscr{F}=\mathscr{F}_{T}$. Recall the Fréchet spaces $\mathbf{C}^{m}$ with semi-norms $\|\cdot\|_{m, C}$ from Section 2.5. As usual, $\mathbf{L}^{0}=\mathbf{L}^{0}(\Omega, \mathscr{F}, \mathbb{P}$ ) denotes the space of (equivalent classes of) random variables with convergence in probability.

Lemma C.1. Let $m$ be a non-negative integer, $U$ be an open set in $\mathbf{R}^{d}$, and $\xi: U \rightarrow \mathbf{L}^{0}$ be a random field with sample paths in $\mathbf{C}^{m}=\mathbf{C}^{m}(U)$ such that for every compact set $C \subset U$

$$
\mathbb{E}\left[\|\xi\|_{m, C}\right]<\infty
$$

Then the stochastic field

$$
M_{t}(x) \triangleq \mathbb{E}\left[\xi(x) \mid \mathscr{F}_{t}\right], \quad t \in[0, T], x \in U,
$$

has a modification with sample paths in $\mathbf{D}\left([0, T], \mathbf{C}^{m}\right)$ and, for every multiindex $k=\left(k_{1}, \ldots, k_{d}\right)$ of non-negative integers with $|k| \triangleq \sum_{i=1}^{d} k_{i} \leq m$,

$$
D^{k} M_{t}(x)=\mathbb{E}\left[D^{k} \xi(x) \mid \mathscr{F}_{t}\right], \quad t \in[0, T], x \in U,
$$

where the differential operator $D^{k}$ is defined in (2.46).

Proof. By induction, it is sufficient to consider the cases $m=0,1$. Assume first that $m=0$. It is well-known that, for every $x \in U$, the martingale $M(x)$ has a modification in $\mathbf{D}([0, T], \mathbf{R})$. Fix a compact set $C \subset U$ and let $\left(x_{i}\right)_{i \geq 1}$ be a dense countable subset of $C$. Standard arguments show that the stochastic field $M: C \times[0, T] \rightarrow \mathbf{R}$ has a modification in $\mathbf{D}([0, T], \mathbf{C})$ if

$$
\begin{aligned}
\lim _{a \rightarrow \infty} \mathbb{P}\left[\sup _{x_{i}}\left(M\left(x_{i}\right)\right)_{T}^{*} \geq a\right] & =0, \\
\lim _{\delta \rightarrow 0} \mathbb{P}\left[\sup _{\left|x_{i}-x_{j}\right| \leq \delta}\left(M\left(x_{i}\right)-M\left(x_{j}\right)\right)_{T}^{*} \geq \varepsilon\right] & =0, \text { for every } \varepsilon>0,
\end{aligned}
$$

where $X_{t}^{*} \triangleq \sup _{0 \leq s \leq t}\left|X_{s}\right|$.

From the conditions on $\xi=\xi(x)$ we deduce that the martingales:

$$
\begin{aligned}
X_{t} & \triangleq \mathbb{E}\left[\sup _{x \in C}|\xi(x)| \mid \mathscr{F}_{t}\right], \\
Y_{t}(\delta) & \triangleq \mathbb{E}\left[\sup _{\left|x_{i}-x_{j}\right| \leq \delta}\left|\xi\left(x_{i}\right)-\xi\left(x_{j}\right)\right| \mid \mathscr{F}_{t}\right],
\end{aligned}
$$


are well-defined and

$$
\lim _{\delta \rightarrow 0} \mathbb{E}\left[Y_{T}(\delta)\right]=0
$$

Since, clearly,

$$
\begin{aligned}
\sup _{x_{i}}\left|M_{t}\left(x_{i}\right)\right| & \leq X_{t}, \\
\sup _{\left|x_{i}-x_{j}\right| \leq \delta}\left|M_{t}\left(x_{i}\right)-M_{t}\left(x_{j}\right)\right| & \leq Y_{t}(\delta),
\end{aligned}
$$

we deduce from Doob's inequality:

$$
\begin{gathered}
\mathbb{P}\left[\sup _{x_{i}}\left(M\left(x_{i}\right)\right)_{T}^{*} \geq a\right] \leq \mathbb{P}\left[X_{T}^{*} \geq a\right] \leq \frac{1}{a} \mathbb{E}\left[X_{T}\right], \\
\mathbb{P}\left[\sup _{\left|x_{i}-x_{j}\right| \leq \delta}\left(M\left(x_{i}\right)-M\left(x_{j}\right)\right)_{T}^{*} \geq \varepsilon\right] \leq \mathbb{P}\left[(Y(\delta))_{T}^{*} \geq \varepsilon\right] \leq \frac{1}{\varepsilon} \mathbb{E}\left[Y_{T}(\delta)\right],
\end{gathered}
$$

which, jointly with (C.3), implies (C.1) and (C.2). This concludes the proof for the case $m=0$.

Assume now that $m=1$ and define the stochastic field

$$
D_{t}(x) \triangleq \mathbb{E}\left[\nabla \xi(x) \mid \mathscr{F}_{t}\right]: U \times[0, T] \rightarrow \mathbf{L}^{0}\left(\mathbf{R}^{d}\right),
$$

with values in $\mathbf{R}^{d}$, where $\nabla \triangleq\left(\frac{\partial}{\partial x^{1}}, \ldots, \frac{\partial}{\partial x^{d}}\right)$ is the gradient operator. From the case $m=0$ we obtain that the stochastic fields $M=M_{t}(x)$ and $D=$ $D_{t}(x)$ have modifications in $\mathbf{D}([0, T], \mathbf{C})$, which we shall use. For $M=$ $M_{t}(x)$ to have a modification in $\mathbf{D}\left([0, T], \mathbf{C}^{1}\right)$ with the derivatives given by $D=D_{t}(x)$ we have to show that

$$
\lim _{\delta \rightarrow 0} \mathbb{P}\left[\sup _{x \in C,|x-y| \leq \delta} \frac{1}{\delta}(N(x, y))_{T}^{*} \geq \varepsilon\right]=0, \text { for every } \varepsilon>0,
$$

where

$$
N(x, y) \triangleq M(y)-M(x)-\langle D(x), y-x\rangle .
$$

We follow the same path as in the proof of the previous case. Our assumptions on $\xi=\xi(x)$ imply that, for sufficiently small $\delta>0$, the martingale

$$
Z_{t}(\delta) \triangleq \mathbb{E}\left[\sup _{x \in C,|x-y| \leq \delta} \frac{1}{\delta}|\xi(y)-\xi(x)-\langle\nabla \xi(x), y-x\rangle| \mid \mathscr{F}_{t}\right]
$$


is well-defined and

$$
\lim _{\delta \rightarrow 0} \mathbb{E}\left[Z_{T}(\delta)\right]=0
$$

Since

$$
\sup _{x \in C,|x-y| \leq \delta} \frac{1}{\delta} N_{t}(x, y) \leq Z_{t}(\delta),
$$

we have, by Doob's inequality,

$$
\mathbb{P}\left[\sup _{x \in C,|x-y| \leq \delta} \frac{1}{\delta}(N(x, y))_{T}^{*} \geq \varepsilon\right] \leq \mathbb{P}\left[(Z(\delta))_{T}^{*} \geq \varepsilon\right] \leq \frac{1}{\varepsilon} \mathbb{E}\left[Z_{T}(\delta)\right],
$$

and (C.4) follows from (C.5).

Lemma C.2. Let $U$ be an open set in $\mathbf{R}^{m}$ (and, in addition, be a convex set for conditions (C3) and $\left(C^{r} 7\right)$ and a cone for $\left(C_{4}\right)$ ). Let furthermore $V$ be an open set in $\mathbf{R}^{l}$, and $\xi=\xi(x, y): U \times V \rightarrow \mathbf{L}^{0}$ be a random field with continuous sample paths such that for every compact set $C \subset U \times V$

$$
\mathbb{E}\left[\sup _{(x, y) \in C}|\xi(x, y)|\right]<\infty .
$$

Then the stochastic field

$$
M_{t}(x, y) \triangleq \mathbb{E}\left[\xi(x, y) \mid \mathscr{F}_{t}\right], \quad 0 \leq t \leq T, x \in U, y \in V,
$$

has a modification with sample paths in $\mathbf{D}([0, T], \mathbf{C}(U \times V))$. Moreover, if the sample paths of $\xi$ belong to $\widetilde{\mathbf{C}}$, then there is a modification of $M$ with sample paths in $\mathbf{D}([0, T], \widetilde{\mathbf{C}})$, where $\widetilde{\mathbf{C}}=\widetilde{\mathbf{C}}(U \times V)$ is any one of the following subspaces of $\mathbf{C}=\mathbf{C}(U \times V)$ :

(C1) $\widetilde{\mathbf{C}}$ consists of all non-negative functions;

(C2) $\widetilde{\mathbf{C}}$ consists of all functions $f=f(x, y)$ which are non-decreasing with respect to $x$;

(C3) $\widetilde{\mathbf{C}}$ consists of all functions $f=f(x, y)$ which are convex with respect to $x$

(C4) $\widetilde{\mathbf{C}}$ consists of all functions $f=f(x, y)$ which are positively homogeneous with respect to $x$ :

$$
f(c x, y)=c f(x, y), \quad c>0
$$


(C5) $\widetilde{\mathbf{C}}$ consists of all strictly positive functions;

(C6) $\widetilde{\mathbf{C}}$ consists of all functions $f=f(x, y)$ which are strictly increasing with respect to $x$ :

$$
f\left(x_{1}, y\right)<f\left(x_{2}, y\right), \quad x_{1} \leq x_{2}, x_{1} \neq x_{2} ;
$$

(C7) $\widetilde{\mathbf{C}}$ consists of all functions $f=f(x, y)$ which are strictly convex with respect to $x$ :

$$
\frac{1}{2}\left(f\left(x_{1}, y\right)+f\left(x_{2}, y\right)\right)>f\left(\frac{1}{2}\left(x_{1}+x_{2}\right), y\right), \quad x_{1} \neq x_{2} .
$$

Proof. The existence of a modification for $M$ with sample paths in $\mathbf{D}([0, T], \mathbf{C})$ has been proved in Lemma C.1. Hereafter we shall use this modification.

The assertions of items $(\mathrm{C} 1)-(\mathrm{C} 4)$ are straightforward, since for every $t \in[0, T]$ these conditions are obviously satisfied for the random field $M_{t}$ : $U \rightarrow \mathbf{L}^{0}$ and the sample paths of $M$ belong to $\mathbf{D}([0, T], \mathbf{C})$.

To verify (C5) recall the well-known fact that if $N$ is a martingale on $[0, T]$ such that $N_{T}>0$, then $\inf _{t \in[0, T]} N_{t}>0$. For every compact set $C \subset U \times V$ we have by (C5) that $\inf _{(x, y) \in C} \xi(x, y)>0$ and, hence,

$$
\inf _{t \in[0, T]} \inf _{(x, y) \in C} M_{t}(x, y) \geq \inf _{t \in[0, T]} \mathbb{E}\left[\inf _{(x, y) \in C} \xi(x, y) \mid \mathscr{F}_{t}\right]>0,
$$

implying (C5). Observe that this argument clearly extends to the case, when $U$ is an $F_{\sigma}$-set, that is, a countable union of closed sets.

The cases (C6) and (C7) follow from (C5) by re-parameterization. For example, to obtain (C6) define the set $\widetilde{U} \subset \mathbf{R}^{2 m}$ and the random fields $\eta: \widetilde{U} \times V \rightarrow \mathbf{L}^{0}$ and $N: \widetilde{U} \times V \times[0, T] \rightarrow \mathbf{L}^{0}$ as

$$
\begin{aligned}
\widetilde{U} & \triangleq\left\{\left(x_{1}, x_{2}\right): x_{i} \in U, x_{1} \leq x_{2}, x_{1} \neq x_{2}\right\}, \\
\eta\left(x_{1}, x_{2}, y\right) & \triangleq \xi\left(x_{2}, y\right)-\xi\left(x_{1}, y\right), \\
N_{t}\left(x_{1}, x_{2}, y\right) & \triangleq M_{t}\left(x_{2}, y\right)-M_{t}\left(x_{1}, y\right) .
\end{aligned}
$$

While the set $\widetilde{U}$ is not open, it is an $F_{\sigma}$-set. An application of (C5) to $\eta$ and $N$ then yields (C6) for $\xi$ and $M$. 
Lemma C.3. In addition to the assumptions of Lemma C.2 suppose that

$$
\mathbb{E}\left[\sup _{(x, y) \in U \times D} \xi(x, y)\right]<\infty,
$$

for every compact set $D \subset V$. Then the assertions of Lemma C.2 also hold for the following subspaces:

(C8) $\widetilde{\mathbf{C}}$ consists of all non-negative functions $f=f(x, y)$ such that for every increasing sequence $\left(C_{n}\right)_{n \geq 1}$ of compact sets in $U$ with $\cup_{n \geq 1} C_{n}=U$ and for every compact set $D \subset V$

$$
\lim _{n \rightarrow \infty} \sup _{x \in U / C_{n}} \sup _{y \in D} f(x, y)=0 ;
$$

(C9) $\widetilde{\mathbf{C}}$ consists of all functions $f=f(x, y)$ such that for every increasing sequence $\left(C_{n}\right)_{n \geq 1}$ of compact sets in $U$ with $\cup_{n \geq 1} C_{n}=U$ and for every compact set $D \subset V$

$$
\lim _{n \rightarrow \infty} \sup _{x \in U / C_{n}} \sup _{y \in D} f(x, y)=-\infty .
$$

Proof. For the proof of (C8) recall that by Doob's inequality, if $\left(N^{n}\right)_{n \geq 1}$ is a sequence of martingales such that $N_{T}^{n} \rightarrow 0$ in $\mathbf{L}^{1}$, then $\left(N^{n}\right)_{T}^{*} \triangleq \sup _{0 \leq t \leq T}\left|N_{t}^{n}\right| \rightarrow$ 0 in $\mathbf{L}^{0}$. Accounting for (C.6) we deduce that, for the compact sets $\left(C_{n}\right)_{n \geq 1}$ and $D$ as in (C8),

$$
\lim _{n \rightarrow \infty} \mathbb{E}\left[\sup _{x \in U / C_{n}} \sup _{y \in D} \xi(x, y)\right]=0 .
$$

The validity of (C8) for the sample paths of $M$ follows now from

$$
\sup _{x \in U / C_{n}} \sup _{y \in D}(M(x, y))_{T}^{*} \leq \sup _{0 \leq t \leq T} \mathbb{E}\left[\sup _{x \in U / C_{n}} \sup _{y \in D} \xi(x, y) \mid \mathscr{F}_{t}\right],
$$

where we used the fact that in $(\mathrm{C} 8) \xi \geq 0$.

Finally, (C9) follows from (C8) if we observe that a function $f=f(x, y)$ satisfies (C9) if and only if for every positive integer $n$ the function

$$
g_{n}(x, y) \triangleq \max (f(x, y)+n, 0), \quad(x, y) \in U \times V,
$$

satisfies (C8).

Acknowledgments. We thank Andreas Hamel for references on the maxrule for subdifferentials used in Appendix A. 


\section{References}

[1] Peter Bank and Dmitry Kramkov. A model for a large investor trading at market indifference prices. I: single-period case. arXiv:1110.3224v2, October 2011. URL http://arxiv.org/abs/1110.3224v2.

[2] Peter Bank and Dmitry Kramkov. A model for a large investor trading at market indifference prices. II: continuous-time case. arXiv:1110.3229v2, October 2011. URL http://arxiv.org/abs/1110.3229v2.

[3] Peter Bank and Dmitry Kramkov. On a stochastic differential equation arising in a price impact model. Stochastic Process. Appl., 123(3):11601175, 2013. ISSN 0304-4149. doi: 10.1016/j.spa.2012.10.011. URL http://dx.doi.org/10.1016/j.spa.2012.10.011.

[4] Rose-Anne Dana. Existence, uniqueness and determinacy of ArrowDebreu equilibria in finance models. J. Math. Econom., 22(6):563579, 1993. ISSN 0304-4068. doi: 10.1016/0304-4068(93)90005-6. URL http://dx.doi .org/10.1016/0304-4068(93)90005-6.

[5] Rose Anne Dana and Cuong Le Van. Asset equilibria in $L^{p}$ spaces with complete markets: a duality approach. J. Math. Econom., 25(3):263280, 1996. ISSN 0304-4068. doi: 10.1016/0304-4068(95)00735-0. URL http://dx.doi.org/10.1016/0304-4068(95)00735-0.

[6] Jean-Baptiste Hiriart-Urruty and Claude Lemaréchal. Fundamentals of convex analysis. Grundlehren Text Editions. Springer-Verlag, Berlin, 2001. ISBN 3-540-42205-6.

[7] Ioannis Karatzas, John P. Lehoczky, and Steven E. Shreve. Existence and uniqueness of multi-agent equilibrium in a stochastic, dynamic consumption/investment model. Math. Oper. Res., 15(1):80128, 1990. ISSN 0364-765X. doi: 10.1287/moor.15.1.80. URL http://dx.doi.org/10.1287/moor.15.1.80.

[8] Hiroshi Kunita. Stochastic flows and stochastic differential equations, volume 24 of Cambridge Studies in Advanced Mathematics. Cambridge University Press, Cambridge, 1990. ISBN 0-521-35050-6.

[9] Paul Milgrom and Ilya Segal. Envelope theorems for arbitrary choice sets. Econometrica, 70(2):583-601, 2002. 
[10] R. Tyrrell Rockafellar. Convex analysis. Princeton Mathematical Series, No. 28. Princeton University Press, Princeton, N.J., 1970.

[11] R. Tyrrell Rockafellar and Roger J.-B. Wets. Variational analysis, volume 317 of Grundlehren der Mathematischen Wissenschaften [Fundamental Principles of Mathematical Sciences]. Springer-Verlag, Berlin, 1998. ISBN 3-540-62772-3. 